\title{
Runs and Patterns in a Sequence of Markov Dependent Bivariate Trials
}

\author{
Kirtee Kiran Kamalja, Ramkrishna Lahu Shinde \\ Department of Statistics, School of Mathematical Sciences, North Maharashtra University, Jalgaon, India \\ E-mail: kirteekamalja@gmail.com \\ Received May 13, 2011; revised June 2, 2011; accepted June 8, 2011
}

\begin{abstract}
In this paper we consider a sequence of Markov dependent bivariate trials whose each component results in an outcome success (0) and failure (1) i.e. we have a sequence $\left\{\left(\begin{array}{l}X_{n} \\ Y_{n}\end{array}\right), n \geq 0\right\}$ of $S=\left\{\left(\begin{array}{l}0 \\ 0\end{array}\right),\left(\begin{array}{l}0 \\ 1\end{array}\right),\left(\begin{array}{l}1 \\ 0\end{array}\right),\left(\begin{array}{l}1 \\ 1\end{array}\right)\right\}$ valued Markov dependent bivariate trials. By using the method of conditional probability generating functions (pgfs), we derive the pgf of joint distribution of $\left(X_{n, k_{0}^{1}}^{0}, X_{n, k_{1}^{1}}^{1} ; Y_{n, k_{0}^{2}}^{0}, Y_{n, k_{1}^{2}}^{1}\right)$ where for $i=0,1, X_{n, k_{i}^{1}}^{i}$ denotes the number of occurrences of $i$-runs of length $k_{i}^{1}$ in the first component and $Y_{n}^{i}$ denotes the number of occurrences of $i$-runs of length $k_{i}^{2}$ in the second component of Markov dependent bivariate trials. Further we consider two patterns $\Lambda_{1}$ and $\Lambda_{2}$ of lengths $k_{1}$ and $k_{2}$ respectively and obtain the pgf of joint distribution of $\left(X_{n, \Lambda_{1}}, Y_{n, \Lambda_{2}}\right)$ using method of conditional probability generating functions where $X_{n, \Lambda_{1}}\left(Y_{n, \Lambda_{2}}\right)$ denotes the number of occurrences of pattern $\Lambda_{1}\left(\Lambda_{2}\right)$ of length $k_{1}\left(k_{2}\right)$ in the first (second) $n$ components of bivariate trials. An algorithm is developed to evaluate the exact probability distributions of the vector random variables from their derived probability generating functions. Further some waiting time distributions are studied using the joint distribution of runs.
\end{abstract}

Keywords: Markov Dependent Bivariate Trials, Conditional Probability Generating Function, Joint Distribution

\section{Introduction}

The distributions of several run statistics are used in various areas such as reliability theory, testing of statistical hypothesis, DNA sequencing, psychology [1], start up demonstration tests [2] etc. There are various counting schemes of runs. Some of the most popular counting schemes of runs are non-overlapping success runs of length $k$ [3], overlapping success runs of length $k$ [4], success runs of length at least $k, \ell$ - overlapping success runs of length $k$ [5], success runs of exact length $k$ [6].

The probability distribution of various run statistics associated with the above counting schemes have been studied extensively in the literature in different situations such as independent Bernoulli trials (BT), non-identical BT, Markov dependent BT (MBT), higher order MBT, binary sequence of order $k$, multi-state trials etc. But very little work is found on the distribution theory of run statistics in case of bivariate trials which has applications in different areas such as start up demonstration tests with regard to simultaneous start ups of two equipment, reliability theory of two dimensional consecutive $(k, r)$ out of $(k+1, n): F$-Lattice system etc as specified by [7]. [7] have studied the distribution of sooner and later waiting time problems for runs in Markov dependent bivariate trials by giving system of linear equations of the conditional pgfs of the waiting times. The distribution of number of occurrences of runs in the two components of bivariate sequence of trials and their joint distributions are still unknown to the literature.

Consider a sequence $\left\{X_{n}, n \geq 0\right\}$ of $S$-valued trials where $S$ is set of all possible outcomes of trials under study. The simple pattern $\Lambda$ is composed of specified sequence of $k$ states i.e. $\Lambda=a_{1} a_{2} \cdots a_{k}$ where $a_{1}, a_{2}, \cdots a_{k} \in S$. The number of occurrences of patterns can be counted according to the non-overlapping or overlapping counting scheme. The non-overlapping counting scheme starts recounting of the pattern immediately after the occurrence of the pattern while the overlapping 
counting scheme of patterns allows an overlap of prespecified fixed length in the successive occurrences of patterns.

Recently the study of distributions of different statistics based on patterns has become a focus area for many researchers due to its wide applicability area. Distribution of $W_{r, k, \Lambda}$, the waiting time for the $r^{\text {th }}$ occurrence of pattern $\Lambda$ of length $k$ in the sequence of multistate trials is studied by $[1,8,9]$. [10] considered the sequence $X_{1}, X_{2}, \cdots$ generated by Polya's urn scheme and study the waiting time distribution of $W_{r, k, \Lambda}$ for $r=1$.

Joint distribution of number of occurrences of pattern $\Lambda_{1}$ of length $k_{1}$ and pattern $\Lambda_{2}$ of length $k_{2}$ in $n$ Markov dependent multi-state trials is studied by [11]. [12] considered a sequence $\underline{X}_{i}, i=1,2, \cdots$ of $m$ dimensional i.i.d. Random column vectors whose entries are $\{0,1\}$-valued i.i.d. random variables and obtain the waiting time distribution of two dimensional patterns with general shape. The general method, which is an extension of method of conditional pgfs, is used to study these distributions by [12].

Even though the distribution of waiting time of the pattern of general shape in the sequence of multi-variate trials with i.i.d. components has been done, the joint distribution of number of occurrences of patterns $\Lambda_{i},(i=1,2, \cdots, m)$ in the sequence of $i^{\text {th }}$ component of the $m$-variate trials $\underline{X}_{1}, \underline{X}_{2}, \cdots, \underline{X}_{n}$ is still unknown. Here we derive the $p g f$ of joint distribution of number of occurrences of runs in both the components of the bivariate trials and generalize this study to the distribution of number of occurrences of patterns in both components of the bivariate trials.

In this paper we consider the sequence $\left\{\left(\begin{array}{l}X_{n} \\ Y_{n}\end{array}\right), n \geq 0\right\}$ of $S$-valued Markov dependent bivariate trials. In Section 2, we obtain the pgf of joint distribution of number of occurrences of $i$-runs of length $k_{i}^{1}$ in first components and $i$-runs of length $k_{i}^{2}$ in the second components of the bivariate trials $(i=0,1)$. We study this joint distribution of runs under the non-overlapping counting scheme of runs by using the method of conditional pgfs. Further in section 3, we study the joint distribution of number of occurrences of pattern $\Lambda_{1}$ of length $k_{1}$ in the first component and number of occurrences of pattern $\Lambda_{2}$ of length $k_{2}$ in the second component of bivariate trials. In Section 4, we develop an algorithm to evaluate the exact probability distributions of the random variables under study. As an application of the derived joint distributions, in Section 5, we obtain distributions of several waiting times associated with the runs and patterns in bivariate trials. In Section 6 we present some numerical work based on distribution of runs and patterns. Finally in Section 7, we discuss an application and generalization of the studied distributions.

\section{The Joint Distribution of Number of Occurrences of Runs}

Let $S=\left\{\left(\begin{array}{l}0 \\ 0\end{array}\right),\left(\begin{array}{l}0 \\ 1\end{array}\right),\left(\begin{array}{l}1 \\ 0\end{array}\right),\left(\begin{array}{l}1 \\ 1\end{array}\right)\right\}$. Consider the sequence $\left\{\left(\begin{array}{l}X_{n} \\ Y_{n}\end{array}\right), n \geq 0\right\}$ of $S$-valued Markov dependent bivariate trials with the transition probabilities,

$$
P\left(\left(\begin{array}{c}
X_{i} \\
Y_{i}
\end{array}\right)=\left(\begin{array}{c}
x \\
y
\end{array}\right) \mid\left(\begin{array}{c}
X_{i-1} \\
Y_{i-1}
\end{array}\right)=\left(\begin{array}{l}
u \\
v
\end{array}\right)\right)=p_{u v, x y} \forall\left(\begin{array}{l}
u \\
v
\end{array}\right),\left(\begin{array}{l}
x \\
y
\end{array}\right) \in S
$$

$i=1,2, \cdots, n$

and the initial probabilities

$P\left(\left(\begin{array}{c}X_{0} \\ Y_{0}\end{array}\right)=\left(\begin{array}{l}x \\ y\end{array}\right)\right)=\pi_{x y}$ for $\left(\begin{array}{l}x \\ y\end{array}\right) \in S$.

Let $X_{n, k_{i}^{1}}^{i}\left(Y_{n, k_{i}^{2}}^{i}\right)$ be the number of $i$-runs $(i=0,1)$ of length $k_{i}^{1}\left(k_{i}^{2}\right)$ in $n$ trials associated with the first (second) component of bivariate trials (i.e. number of $i$-runs in $\left.X_{0}, X_{1}, \cdots, X_{n}\left(Y_{0}, Y_{1}, \cdots, Y_{n}\right)\right)$. In this section we derive the joint distribution of

$\left(X_{n, k_{0}^{1}}^{0}, X_{n, k_{1}^{1}}^{1} ; Y_{n, k_{0}^{2}}^{0}, Y_{n, k_{1}^{2}}^{1}\right)$.

Let $\phi_{n}\left(t_{0}, t_{1} ; s_{0}, s_{1}\right)$ be the $p g f$ of distribution of $\left(X_{n, k_{0}^{1}}^{0}, X_{n, k_{1}^{1}}^{1} ; Y_{n, k_{0}^{2}}^{0}, Y_{n, k_{1}^{2}}^{1}\right)$. Assume that for a non-negative integer $c \leq n$, we have observed until $(n-c)^{\text {th }}$ trial (i.e. we have observed $\left.\left(\begin{array}{c}X_{i} \\ Y_{i}\end{array}\right), i=0,1, \cdots, n-c\right)$. We define $\phi_{c}^{\left(i, m_{i}^{1} ; j, m_{j}^{2}\right)}=\phi_{c}^{\left(i, m_{i}^{1} ; j, m_{j}^{2}\right)}\left(t_{0}, t_{1} ; s_{0}, s_{1}\right)$ as pgf of conditional distribution of number of $i$-runs of length $k_{i}^{1}$ in $X_{n-c+1}, \cdots, X_{n}$ and number of $j$-runs of length $k_{j}^{2}$ in $Y_{n-c+1}, \cdots, Y_{n}$ given that we have observed

$\left(\begin{array}{c}X_{0} \\ Y_{0}\end{array}\right),\left(\begin{array}{c}X_{1} \\ Y_{1}\end{array}\right), \cdots,\left(\begin{array}{c}X_{n-c} \\ Y_{n-c}\end{array}\right)$ and currently have $i$-run of length $m_{i}^{1}$ in first component and $j$-runs of length $m_{j}^{2}$ in the second component of bivariate trials is observed, $m_{i}^{1}=1,2, \cdots, k_{i}^{1}, m_{j}^{2}=1,2, \cdots, k_{j}^{2}, i, j=0,1$.

We define $a^{c}=1-a$ for $a=0,1$.

Now by assuming $\pi_{00}=1$, we have,

$$
\phi_{n}\left(t_{0}, t_{1} ; s_{0}, s_{1}\right)=\phi_{n}^{(0,0 ; 0,0)}\left(t_{0}, t_{1} ; s_{0}, s_{1}\right)
$$

Also we have,

$$
\begin{aligned}
& \phi_{0}^{\left(i, m_{i}^{1} ; j, m_{j}^{2}\right)}\left(t_{0}, t_{1} ; s_{0}, s_{1}\right)=1 \text { for } m_{i}^{1}=1,2, \ldots, k_{i}^{1}, \\
& m_{j}^{2}=1,2, \ldots, k_{j}^{2} \text { and } i, j=0,1
\end{aligned}
$$

Conditioning on the first trial we have the following system of recurrent relation of conditional pgfs for 
$c=1,2, \cdots, n$.

$$
\begin{aligned}
& \phi_{n}^{(0,0 ; 0,0)}\left(t_{0}, t_{1} ; s_{0}, s_{1}\right) \\
& =p_{00,00} \phi_{n-1}^{(0,1 ; 0,1)}+p_{00,01} \phi_{n-1}^{(0,1 ; 1,1)} \\
& +p_{00,10} \phi_{n-1}^{(1,1 ; 0,1)}+p_{00,11} \phi_{n-1}^{(1,1 ; 1,1)}
\end{aligned}
$$

Conditioning on the next trial from each stage, we have the following system of recurrent relations of conditional pgfs for $c=1,2, \cdots, n$ and $i, j=0,1$.

$$
\begin{aligned}
& \phi_{c}^{\left(i, m_{i}^{1} ; j, m_{j}^{2}\right)} \\
& =p_{i j, i j} \phi_{c-1}^{\left(i, m_{i}^{1}+1 ; j, m_{j}^{2}+1\right)}+p_{i j, i^{c} j} \phi_{c-1}^{\left(i^{c}, 1 ; j, m_{j}^{2}+1\right)} \\
& +p_{i j, i j^{c}} \phi_{c-1}^{\left(i, m_{i}^{1}+1 ; j^{c}, 1\right)}+p_{i j, i^{c} j^{c}} \phi_{c-1}^{\left(i^{c}, 1 ; j^{c}, 1\right)} \\
& \text { if } m_{i}^{1}=1,2, \cdots, k_{i}^{1}-2, \quad m_{j}^{2}=1,2, \cdots, k_{j}^{2}-2 \text {, } \\
& \phi_{c}^{\left(i, m_{i}^{1} ; j, m_{j}^{2}\right)} \\
& =p_{i j, i j} \phi_{c-1}^{\left(i, m_{i}^{1}+1 ; j, m_{j}^{2}+1\right)} t_{i}+p_{i j, i^{c} j} \phi_{c-1}^{\left(i^{c}, 1 ; j, m_{j}^{2}+1\right)} \\
& +p_{i j, i j} \phi_{c-1}^{\left(i, m_{i}^{1}+1 ; j^{c}, 1\right)} t_{i}+p_{i j, i^{c} j^{c}} \phi_{c-1}^{\left(i^{c}, 1 ; j^{c}, 1\right)} \\
& \text { if } m_{i}^{1}=k_{i}^{1}-1, m_{j}^{2}=1,2, \cdots, k_{j}^{2}-2 \text {, } \\
& \phi_{c}^{\left(i, m_{i}^{1} ; j, m_{j}^{2}\right)}=p_{i j, i j} \phi_{c-1}^{\left(i, 1 ; j, m_{j}^{2}+1\right)}+p_{i j, i^{c} j} \phi_{c-1}^{\left(i^{c}, 1 ; j, m_{j}^{2}+1\right)} \\
& +p_{i j, i j} \phi_{c-1}^{\left(i, 1 ; j^{c}, 1\right)}+p_{i j, i^{c} j^{c}} \phi_{c-1}^{\left(i^{c}, 1 ; j^{c}, 1\right)} \\
& \text { if } m_{i}^{1}=k_{i}^{1}, \quad m_{j}^{2}=1,2, \cdots, k_{j}^{2}-2 \\
& \phi_{c}^{\left(i, m_{i}^{1} ; j, m_{j}^{2}\right)}=p_{i j, i j} \phi_{c-1}^{\left(i, m_{i}^{1}+1 ; j, m_{j}^{2}+1\right)} s_{j}+p_{i j, i^{c} j} \phi_{c-1}^{\left(i^{c}, 1 ; j, m_{j}^{2}+1\right)} s_{j} \\
& +p_{i j, i j^{c}} \phi_{c-1}^{\left(i, m_{i}^{1}+1 ; j^{c}, 1\right)}+p_{i j, i^{c} j^{c}} \phi_{c-1}^{\left(i^{c}, 1 ; j^{c}, 1\right)} \\
& \text { if } m_{i}^{1}=1,2, \cdots, k_{i}^{1}-2, m_{j}^{2}=k_{j}^{2}-1 \text {, } \\
& \phi_{c}^{\left(i, m_{i}^{1} ; j, m_{j}^{2}\right)}=p_{i j, i j} \phi_{c-1}^{\left(i, m_{i}^{1}+1 ; j, m_{j}^{2}+1\right)} t_{i} S_{j}+p_{i j, i^{c} j} \phi_{c-1}^{\left(i^{c}, 1 ; j, m_{j}^{2}+1\right)} S_{j} \\
& +p_{i j, i j^{c}} \phi_{c-1}^{\left(i, m_{i}^{1}+1 ; j^{c}, 1\right)} t_{i}+p_{i j, i^{c} j^{c}} \phi_{c-1}^{\left(i^{c}, 1 ; j^{c}, 1\right)} \\
& \text { if } m_{i}^{1}=k_{i}^{1}-1, m_{j}^{2}=k_{j}^{2}-1, \\
& \phi_{c}^{\left(i, m_{i}^{1} ; j, m_{j}^{2}\right)}=p_{i j, i j} \phi_{c-1}^{\left(i, 1, j, m_{j}^{2}+1\right)} s_{j}+p_{i j, i^{c} j} \phi_{c-1}^{\left(i^{c}, 1 ; j, m_{j}^{2}+1\right)} S_{j} \\
& +p_{i j, i j^{c}} \phi_{c-1}^{\left(i, 1 ; j^{c}, 1\right)}+p_{i j, i^{c} j^{c}} \phi_{c-1}^{\left(i^{c}, 1 ; j^{c}, 1\right)} \\
& \text { if } m_{i}^{1}=k_{i}^{1}, m_{j}^{2}=k_{j}^{2}-1, \\
& \phi_{c}^{\left(i, m_{i}^{1} ; j, m_{j}^{2}\right)}=p_{i j, i j} \phi_{c-1}^{\left(i, m_{i}^{1}+1 ; j, 1\right)}+p_{i j, i^{c} j} \phi_{c-1}^{\left(i^{c}, 1 ; j, 1\right)} \\
& +p_{i j, i j^{c}} \phi_{c-1}^{\left(i, m_{i}^{1}+1 ; j^{c}, 1\right)}+p_{i j, i^{c} j^{c}} \phi_{c-1}^{\left(i^{c}, 1 ; j^{c}, 1\right)} \\
& \text { if } m_{i}^{1}=1,2, \cdots, k_{i}^{1}-2, m_{j}^{2}=k_{j}^{2} \text {, }
\end{aligned}
$$

$$
\begin{gathered}
\phi_{c}^{\left(i, m_{i}^{1} ; j, m_{j}^{2}\right)}=p_{i j, i j} \phi_{c-1}^{\left(i, m_{i}^{1}+1 ; j, 1\right)} t_{i}+p_{i j, i^{c} j} \phi_{c-1}^{\left(i^{c}, 1 ; j, 1\right)} \\
+p_{i j, i j^{c}} \phi_{c-1}^{\left(i, m_{i}^{1}+1 ; j^{c}, 1\right)} t_{i}+p_{i j, i^{c} j^{c}} \phi_{c-1}^{\left(i^{c}, 1 ; j^{c}, 1\right)} \\
\text { if } m_{i}^{1}=k_{i}^{1}-1, m_{j}^{2}=k_{j}^{2} \\
\phi_{c}^{\left(i, m_{i}^{1} ; j, m_{j}^{2}\right)}=p_{i j, i j} \phi_{c-1}^{(i, 1 ; j, 1)}+p_{i j, i^{c} j} \phi_{c-1}^{\left(i^{c}, 1 ; j, 1\right)} \\
+p_{i j, i j^{c}} \phi_{c-1}^{\left(i, 1 ; j^{c}, 1\right)}+p_{i j, i^{c} j^{c}} \phi_{c-1}^{\left(i^{c}, 1 ; j^{c}, 1\right)} \\
\quad \text { if } m_{i}^{1}=k_{i}^{1}, \quad m_{j}^{2}=k_{j}^{2}
\end{gathered}
$$

Thus we have $\left(k_{0}^{1}+k_{1}^{1}\right)\left(k_{0}^{2}+k_{1}^{2}\right)$ recurrent relations of conditional pgfs for $c=1,2, \cdots, n-1$ and these can be written as,

$$
\begin{aligned}
& \underline{\phi}_{c}\left(t_{0}, t_{1} ; s_{0}, s_{1}\right)= \\
& \left(A+\sum_{i=0}^{1} B_{i}^{1} t_{i}+\sum_{j=0}^{1} B_{j}^{2} s_{j}+\sum_{i=0}^{1} \sum_{j=0}^{1} B_{i j}^{12} t_{i} s_{j}\right) \underline{\phi}_{c-1}\left(t_{0}, t_{1} ; s_{0}, s_{1}\right)
\end{aligned}
$$

where

$$
\begin{aligned}
& \underline{\phi}_{c}=\left(\begin{array}{llllll}
\phi_{c}^{(0,0 ; 0,0)} & \phi_{c}^{(0,1 ; 0,1)} & \cdots & \phi_{c}^{\left(0,1 ; 0, k_{0}^{2}\right)} & \phi_{c}^{(0,1 ; 1,1)} & \cdots
\end{array}\right. \\
& \left.\phi_{c}^{\left(0,1 ; 1, k_{1}^{2}\right)} \quad \cdots \quad \phi_{c}^{\left(1,1 ; 1, k_{1}^{2}\right)} \quad \cdots \phi_{c}^{\left(1, k_{1}^{1} ; 1, k_{1}^{2}\right)}\right)^{\prime}
\end{aligned}
$$

and $A+\sum_{i=0}^{1} B_{i}^{1} t_{i}+\sum_{j=0}^{1} B_{j}^{2} s_{j}+\sum_{i=0}^{1} \sum_{j=0}^{1} B_{i j}^{12} t_{i} s_{j} \quad$ is a square matrix of size $\left(k_{0}^{1}+k_{1}^{1}\right)\left(k_{0}^{2}+k_{1}^{2}\right)$. Each row of this matrix corresponds to corresponding element of $\underline{\phi}_{c}\left(t_{0}, t_{1} ; s_{0}, s_{1}\right)$ and its elements are the coefficients of elements of $\underline{\phi}_{c-1}\left(t_{0}, t_{1} ; s_{0}, s_{1}\right)$.

For $c=0$, we have,

$\underline{\phi}_{0}\left(t_{0}, t_{1} ; s_{0}, s_{1}\right)=\underline{1}$

where $\underline{1}$ is the column vector with all the elements equal to 1 .

Using (2.3) recurrently for $c=1,2, \cdots, n$, we have,

$$
\begin{aligned}
& \underline{\phi}_{n}\left(t_{0}, t_{1} ; s_{0}, s_{1}\right) \\
& =\left(A+\sum_{i=0}^{1} B_{i}^{1} t_{i}+\sum_{j=0}^{1} B_{j}^{2} s_{j}+\sum_{i=0}^{1} \sum_{j=0}^{1} B_{i j}^{12} t_{i} s_{j}\right)^{n} \underline{\phi}_{0}\left(t_{0}, t_{1} ; s_{0}, s_{1}\right)
\end{aligned}
$$

Hence from (2.1) and (2.4) we get the pgf of distribution of $\left(X_{n, k_{0}^{1}}^{0}, X_{n, k_{1}^{1}}^{1} ; Y_{n, k_{0}^{2}}^{0}, Y_{n, k_{1}^{2}}^{1}\right)$ as,

$$
\begin{aligned}
& \phi_{n}\left(t_{0}, t_{1} ; s_{0}, s_{1}\right) \\
& =\underline{p^{\prime}}\left(A+\sum_{i=0}^{1} B_{i}^{1} t_{i}+\sum_{j=0}^{1} B_{j}^{2} s_{j}+\sum_{i=0}^{1} \sum_{j=0}^{1} B_{i j}^{12} t_{i} s_{j}\right)^{n} \underline{1}
\end{aligned}
$$


where $p$ is the first column of identity matrix of order $\left(k_{0}^{1}+k_{1}^{1}\right)\left(k_{0}^{2}+k_{1}^{2}\right)$.

\section{The Joint Distribution of Number of Occurrences of Patterns}

Let $\Lambda_{1}=a_{1} a_{2} \cdots a_{k_{1}}$ and $\Lambda_{2}=b_{1} b_{2} \cdots b_{k_{2}}$ be two patterns of lengths $k_{1}$ and $k_{2}$ respectively where $a_{i}, b_{j} \in\{0,1\}, i=1,2, \cdots, k_{1} ; j=1,2, \cdots, k_{2}$. We consider the non-overlapping counting scheme of patterns in which to count the number of occurrences of pattern $\Lambda$ in a given sequence of $n$ trials, one has to restart counting from scratch each time the pattern $\Lambda$ occurs. Let $X_{n, \Lambda_{1}}$ denotes the number of non-overlapping occurrences of patterns $\Lambda_{1}$ in the first component of $n$ bivariate trials (i.e. in $X_{1}, X_{2}, \cdots, X_{n}$ ) and $Y_{n, \Lambda_{2}}$ denotes the number of non-overlapping occurrences of patterns $\Lambda_{2}$ in the second component of $n$ bivariate trials (i.e. in $\left.Y_{1}, Y_{2}, \cdots, Y_{n}\right)$. Consider the random vector $\left(X_{n, \Lambda_{1}}, Y_{n, \Lambda_{2}}\right)$. In this section we obtain the $p g f$ of joint distribution of $\left(X_{n, \Lambda_{1}}, Y_{n, \Lambda_{2}}\right)$ using method of conditional pgfs.

Let $p g f$ of joint distribution of $\left(X_{n, \Lambda_{1}}, Y_{n, \Lambda_{2}}\right)$ be $\phi_{n}\left(t_{1}, t_{2}\right)=\phi_{n}(\underline{t})$. Assume that for a non-negative integer $c \leq n$, we have observed until $(n-c)^{\text {th }}$ trial i.e. $\left(\begin{array}{c}X_{1} \\ Y_{1}\end{array}\right),\left(\begin{array}{c}X_{2} \\ Y_{2}\end{array}\right), \cdots,\left(\begin{array}{c}X_{n-c} \\ Y_{n-c}\end{array}\right)$. For $c \leq n$, we define, the following conditional $p g f \mathrm{~s}$.

Let $\phi_{c}^{(i, j)}(\underline{t})$ be $p g f$ of conditional distribution of number of occurrences of patterns $\Lambda_{1}$ in $X_{n-c+1}, \cdots, X_{n}$ and number of occurrences of pattern $\Lambda_{2}$ in $Y_{n-c+1}, \cdots, Y_{n}$ of bivariate trials given that currently (i.e. at $(n-c)^{t t^{n}}$ trial) we have observed none of the subpatterns of $\Lambda_{1}$ and $\Lambda_{2}$ and $\left(\begin{array}{c}X_{n-c} \\ Y_{n-c}\end{array}\right)=\left(\begin{array}{l}i \\ j\end{array}\right)$, where $\left(\begin{array}{l}i \\ j\end{array}\right) \in S$.

Similarly let, $\phi_{c, \Lambda_{1}}^{(i, j)}(\underline{t})$ be pgf of conditional distribution of number of occurrences of patterns $\Lambda_{1}$ in $X_{n-c+1}, \cdots, X_{n}$ and patterns $\Lambda_{2}$ in $Y_{n-c+1}, \cdots, Y_{n}$ of bivariate trials given that at $(n-c)^{\text {th }}$ trial we have observed the sub-pattern of length $i$ of $\Lambda_{1}$ in the first component and none of the sub pattern of $\Lambda_{2}$ is observed in the second component of bivariate trials and $\left(\begin{array}{c}X_{n-c} \\ Y_{n-c}\end{array}\right)=\left(\begin{array}{c}a_{i} \\ j\end{array}\right)$ where $i=1,2, \cdots, k_{1}$ and $j=0,1$.

Let $\phi_{c, \Lambda_{2}}^{(i, j)}(\underline{t})$ be pgf of conditional distribution of number of occurrences of patterns $\Lambda_{1}$ in $X_{n-c+1}, \cdots, X_{n}$ and patterns $\Lambda_{2}$ in $Y_{n-c+1}, \cdots, Y_{n}$ of bivariate trials given that at $(n-c)^{\text {th }}$ trial we have observed none of the sub-pattern of $\Lambda_{1}$ in the first component and a sub pattern of the length $j$ of $\Lambda_{2}$ in the second component of bivariate trials is observed and $\left(\begin{array}{c}X_{n-c} \\ Y_{n-c}\end{array}\right)=\left(\begin{array}{c}i \\ b_{j}\end{array}\right)$ where $i=0,1$ and $j=1,2, \cdots, k_{2}$.

Also let $\phi_{c, \Lambda_{1}, \Lambda_{2}}^{(i, j)}(\underline{t})$ as pgf of conditional distribution of number of occurrences of pattern $\Lambda_{1}$ in $X_{n-c+1}, \cdots, X_{n}$ and pattern $\Lambda_{2}$ in $Y_{n-c+1}, \cdots, Y_{n}$ of bivariate trials given that at $(n-c)^{\text {th }}$ trial we have observed the sub-pattern of length $i$ of $\Lambda_{1}$ in the first component and a sub pattern of the length $j$ of $\Lambda_{2}$ in the second component of bivariate trials and $\left(\begin{array}{l}X_{n-c} \\ Y_{n-c}\end{array}\right)=\left(\begin{array}{l}a_{i} \\ b_{j}\end{array}\right)$ where $i=1,2, \cdots, k_{1}$ and $j=1,2, \cdots, k_{2}$.

Let,

$a_{i}^{c}=1-a_{i}, \quad i=1,2, \cdots, k_{1}$

and $b_{j}^{c}=1-b_{j}, \quad j=1,2, \cdots, k_{2}$.

For $c \leq n$, we assume that at $(n-c)^{\text {th }}$ trial, the sub-pattern of length $i$ of $\Lambda_{1}$ is observed in the first component of bivariate trials. If we condition on the next trial as $\left(\begin{array}{l}X_{n-c+1} \\ Y_{n-c+1}\end{array}\right)=\left(\begin{array}{l}a_{i+1}^{c} \\ y_{n-c+1}\end{array}\right)$ i.e. the sub-pattern of length $i$ of $\Lambda_{1}$ observed at $(n-c)^{\text {th }}$ trial breaks at $(n-c+1)^{s t}$ trial then to check whether any sub-pattern of $\Lambda_{1}$ of length $r(r \leq i)$ has occurred, we define the indicator function $\varepsilon_{i r}^{1}$ as,

$$
\begin{gathered}
\varepsilon_{i r}^{1}=I\left\{a_{1}=a_{i-r+2}, a_{2}=a_{i-r+3}, \ldots, a_{r-1}=a_{i}, a_{r}=a_{i+1}^{c}\right\} \\
i=1,2, \cdots, k_{1}-1 ; r \leq i .
\end{gathered}
$$

Similarly we have indicator function $\varepsilon_{j r}^{2}$ as,

$$
\begin{gathered}
\varepsilon_{j r}^{2}=I\left\{b_{1}=b_{j-r+2}, b_{2}=b_{j-r+3}, \ldots, b_{r-1}=b_{j}, b_{r}=b_{j+1}^{c}\right\} \\
j=1,2, \cdots, k_{2}-1 ; r \leq j .
\end{gathered}
$$

Let

$$
\begin{gathered}
u_{1}^{i}=\max _{1 \leq r \leq i} \varepsilon_{i r}^{1}, u_{2}^{i}=\max \left\{r \mid \varepsilon_{i r}^{1}=1, r=1,2, \cdots, i\right\}, \\
i=1,2, \cdots, k_{1}-1 \\
v_{1}^{j}=\max _{1 \leq r \leq j} \varepsilon_{j r}^{2} \text { and } v_{2}^{j}=\max \left\{r \mid \varepsilon_{j r}^{2}=1, r=1,2, \cdots, j\right\} \\
j=1,2, \cdots, k_{2}-1
\end{gathered}
$$

Assuming $\pi_{00}=1$, we have,

$$
\phi_{n}\left(t_{1}, t_{2}\right)=\phi_{n}^{(0,0)}\left(t_{1}, t_{2}\right)
$$

We also have,

$$
\phi_{0}^{(i, j)}(\underline{t})=1 \quad \text { for } \forall\left(\begin{array}{l}
i \\
j
\end{array}\right) \in S ;
$$


$\phi_{0, \Lambda_{1}}^{(i, j)}(\underline{t})=1 \quad$ for $i=1,2, \cdots, k_{1}$ and $j=0,1$

$$
\phi_{0, \Lambda_{2}}^{(i, j)}(\underline{t})=1 \quad \text { for } i=0,1 \text { and } j=1,2, \cdots, k_{2} ;
$$

$\phi_{0, \Lambda_{1}, \Lambda_{2}}^{(i, j)}(\underline{t})=1 \quad$ for $i=1,2, \cdots, k_{1}$ and $j=1,2, \cdots, k_{2}$.

Now for each $1 \leq c \leq n$ ( $c$ denotes the number of trials remaining to observe) we condition on the next trial to obtain the recurrent relations of conditional $p g f s$ as follows.

$$
\begin{aligned}
& \phi_{c}^{(i, j)}(\underline{t})=p_{i j, a_{1} b_{1}} \phi_{c-1, \Lambda_{1}, \Lambda_{2}}^{(1,1)}(\underline{t})+p_{i j, a_{1}^{c} b_{1}} \phi_{c-1, \Lambda_{2}}^{\left(a_{1}^{c}, 1\right)}(\underline{t}) \\
& +p_{i j, a_{1} b_{1}^{b}} \phi_{c-1, \Lambda_{1}}^{\left(1, b_{1}^{c}\right)}(\underline{t})+p_{i j, a_{1}^{c} b_{1}^{c}} \phi_{c-1}^{\left(a_{1}^{c}, b_{1}^{c}\right)}(\underline{t}) \\
& \text { if } i, j=0,1 \text {, } \\
& \phi_{c, \Lambda_{1}}^{(i, j)}(\underline{t})=p_{a_{i} j, a_{i+1} b_{1}} \phi_{c-1, \Lambda_{1}, \Lambda_{2}}^{(i+1,1)}(\underline{t}) \\
& +p_{a_{i} j, a_{i+1}^{c} b_{1}}\left(\phi_{c-1, \Lambda_{1}, \Lambda_{2}}^{\left(u_{i}^{i}, 1\right)}(\underline{t}) u_{1}^{i}+\phi_{c-1, \Lambda_{2}}^{\left(a_{i+1}^{c}, 1\right)}(\underline{t})\left(1-u_{1}^{i}\right)\right) \\
& +p_{a_{i} j, a_{i+1}^{c} b_{1}^{c}}\left(\phi_{c-1, \Lambda_{1}}^{\left(u_{i}^{i}, b_{1}^{c}\right)}(\underline{t}) u_{1}^{i}+\phi_{c-1}^{\left(a_{i+1}^{c}, b_{1}^{c}\right)}(\underline{t})\left(1-u_{1}^{i}\right)\right) \\
& +p_{a_{i} j, a_{i+1} b_{1}^{c}} \phi_{c-1, \Lambda_{1}}^{\left(i+1, b_{1}^{c}\right)}(\underline{t}) \\
& \text { if } i=1,2, \cdots, k_{1}-2 ; j=0,1 \\
& \phi_{c, \Lambda_{1}}^{(i, j)}(\underline{t})=p_{a_{i} j, a_{i+1} b_{1}} \phi_{c-1, \Lambda_{1}, \Lambda_{2}}^{(i+1,1)}(\underline{t}) t_{1} \\
& +p_{a_{i} j, a_{i+1}^{c} b_{1}}\left(\phi_{c-1, \Lambda_{2}}^{\left(u_{2}^{i}, 1\right)}(\underline{t}) u_{1}^{i}+\phi_{c-1, \Lambda_{2}}^{\left(a_{i+1}^{c}, 1\right)}(\underline{t})\left(1-u_{1}^{i}\right)\right) \\
& +p_{a_{i} j, a_{i+1}^{c} b_{1}^{c}}\left(\phi_{c-1, \Lambda_{2}}^{\left(u_{2}^{i}, b_{2}^{c}\right)}(\underline{t}) u_{1}^{i}+\phi_{c-1}^{\left(a_{i+1}^{c}, b_{1}^{c}\right)}(\underline{t})\left(1-u_{1}^{i}\right)\right) \\
& +p_{a_{i} j, a_{i+1} c_{1}} \phi_{c-1, \Lambda_{1}}^{\left(i+1, b_{1}^{c}\right)}(\underline{t}) t_{1} \\
& \text { if } \quad i=k_{1}-1 ; j=0,1 \\
& \phi_{c, \Lambda_{1}}^{(i, j)}(\underline{t})=p_{a_{i} j, a_{1} b_{1}} \phi_{c-1, \Lambda_{1}, \Lambda_{2}}^{(1,1)}(\underline{t})+p_{a_{i} j, a_{1}^{c} b_{1}} \phi_{c-1, \Lambda_{2}}^{\left(a_{c}^{c}, 1\right)}(\underline{t}) \\
& \left.+p_{a_{i} j, a_{1} c} \phi_{1}^{\left(1, b_{1}^{c}\right)}(t) \Lambda_{1}\right)+p_{a_{i} j, a_{1}^{c} b_{1}^{c}} \phi_{c-1}^{\left(a_{1}^{c}, b_{1}^{c}\right)}(\underline{t}) \\
& \text { if } i=k_{1}, j=0,1 \text {. }
\end{aligned}
$$

Similarly we have recurrent relations of the conditional $p g f \mathrm{~s} \quad \phi_{c, \Lambda_{2}}^{(i, j)}(\underline{t})$ for $i=0,1$ and $j=1,2, \cdots, k_{2}$ as follows.

$$
\begin{aligned}
\phi_{c, \Lambda_{2}}^{(i, j)}(\underline{t}) & =p_{i b_{j}, a_{1} b_{j+1}} \phi_{c-1, \Lambda_{1}, \Lambda_{2}}^{(1, j+1)}(\underline{t}) \\
& +p_{i b_{j}, a_{1} b_{j+1}^{c}}\left(\phi_{c-1, \Lambda_{1}, \Lambda_{2}}^{\left(1, v_{j}^{j}\right)}(\underline{t}) v_{1}^{j}+\phi_{c-1, \Lambda_{1}}^{\left(1, b_{j+1}^{c}\right)}(\underline{t})\left(1-v_{1}^{j}\right)\right) \\
& +p_{i b_{j}, a_{1}^{c} b_{j+1}^{c}}\left(\phi_{c-1, \Lambda_{2}}^{\left(a_{1}^{c}, v_{2}^{j}\right)}(\underline{t}) v_{1}^{j}+\phi_{c-1}^{\left(a_{1}^{c}, b_{j+1}^{c}\right)}(\underline{t})\left(1-v_{1}^{j}\right)\right) \\
& +p_{i b_{j}, a_{1}^{c} b_{j+1}} \phi_{c-1, \Lambda_{2}}^{\left(a_{1}^{c}, j+1\right)}(\underline{t})
\end{aligned}
$$$$
\text { if } j=1,2, \cdots, k_{2}-2 \text {; }
$$

$$
\begin{aligned}
& \phi_{c, \Lambda_{2}}^{(i, j)}(\underline{t})=p_{i b_{j}, a_{1} b_{j+1}} \phi_{c-1, \Lambda_{1}, \Lambda_{2}}^{(1, j+1)}(\underline{t}) t_{2} \\
& \quad+p_{i b_{j}, a_{1} b_{j+1}^{c}}\left(\phi_{c-1, \Lambda_{1}, \Lambda_{2}}^{\left(1,, v_{2}^{j}\right)}(\underline{t}) v_{1}^{j}+\phi_{c-1, \Lambda_{1}}^{\left(1, b_{b j}^{c}\right)}(\underline{t})\left(1-v_{1}^{j}\right)\right) \\
& \quad+p_{i b_{j}, a_{1}^{c} b_{j+1}^{c}}\left(\phi_{c-1, \Lambda_{2}}^{\left(a_{1}^{c}, v_{2}^{j}\right)}(\underline{t}) v_{1}^{j}+\phi_{c-1}^{\left(a_{1}^{c}, b_{j+1}^{c}\right)}(\underline{t})\left(1-v_{1}^{j}\right)\right) \\
& \quad+p_{i b_{j}, a_{1}^{c} b_{j+1}} \phi_{c-1, \Lambda_{2}}^{\left(a_{1}^{c}, j+1\right)}(\underline{t}) t_{2}
\end{aligned}
$$

if $j=k_{2}-1$;

$$
\begin{aligned}
\phi_{c, \Lambda_{2}}^{(i, j)}(\underline{t})= & p_{i b_{j}, a_{1} b_{1}} \phi_{c-1, \Lambda_{1}, \Lambda_{2}}^{(1,1)}(\underline{t})+p_{i b_{j}, a_{1}^{c} b_{1}} \phi_{c-1, \Lambda_{2}}^{\left(a_{a}^{c}, 1\right)}(\underline{t}) \\
& +p_{i b_{j}, a_{1} b_{1}^{c}} \phi_{c-1, \Lambda_{1}}^{\left(1, b_{1}^{c}\right)}(\underline{t})+p_{i b_{j}, a_{1}^{c} b_{1}^{c}} \phi_{c-1}^{\left(a_{c}^{c}, b_{1}^{c}\right)}(\underline{t})
\end{aligned}
$$

if $j=k_{2}$

The recurrent relations of the conditional $p g f \mathrm{~s}$ $\phi_{c, \Lambda_{1}, \Lambda_{2}}^{(i, j)}(\underline{t})$ for $i=1,2, \cdots, k_{1}$ and $j=1,2, \cdots, k_{2}$ are as follows.

$$
\begin{aligned}
& \phi_{c, \Lambda_{1}, \Lambda_{2}}^{(i, j)}(\underline{t})=p_{a_{i} b_{j}, a_{i+1} b_{j+1}} \phi_{c-1, \Lambda_{1}, \Lambda_{2}}^{(i+1, j+1)}(\underline{t}) \\
& +p_{a_{i} b_{j}, a_{i+1} b_{j+1}^{c}}\left(\phi_{c-1, \Lambda_{1}, \Lambda_{2}}^{\left(i+1, v_{j}^{j}\right)}(\underline{t}) v_{1}^{j}+\phi_{c-1, \Lambda_{1}}^{\left(i+1, b_{j+1}^{c}\right)}(\underline{t})\left(1-v_{1}^{j}\right)\right) \\
& +p_{a_{i} b_{j}, a_{i+1}^{c} b_{j+1}}\left(\phi_{c-1, \Lambda_{1}, \Lambda_{2}}^{\left(u_{i}^{i}, j+1\right)}(\underline{t}) u_{1}^{i}+\phi_{c-1, \Lambda_{1}}^{\left(a_{i+1}^{c}, j+1\right)}(\underline{t})\left(1-u_{1}^{i}\right)\right) \\
& +p_{a_{i} b_{j}, a_{i+1}^{c} b b_{j+1}^{c}}\left(\phi_{c-1, \Lambda_{1}, \Lambda_{2}}^{\left(u_{i}^{i}, v_{j}^{j}\right)}(\underline{t}) u_{1}^{i} v_{1}^{j}\right. \\
& +\phi_{c-1, \Lambda_{1}}^{\left(u_{i}^{i}, b_{j+1}^{c}\right)}(\underline{t}) u_{1}^{i}\left(1-v_{1}^{j}\right) \\
& +\phi_{c-1, \Lambda_{2}}^{\left(a_{i+1}^{c}, v_{2}^{j}\right)}(\underline{t})\left(1-u_{1}^{i}\right) v_{1}^{j} \\
& \left.+\phi_{c-1}^{\left(a_{i+1}^{c}, b_{j+1}^{c}\right)}(\underline{t})\left(1-u_{1}^{i}\right)\left(1-v_{1}^{j}\right)\right) \\
& \text { if } i=1,2, \cdots, k_{1}-2 ; j=1,2, \cdots, k_{2}-2 \\
& \phi_{c, \Lambda_{1}, \Lambda_{2}}^{(i, j)}(\underline{t})=p_{a_{i} b_{j}, a_{i+1} b_{j+1}} \phi_{c-1, \Lambda_{1}, \Lambda_{2}}^{(i+1, j+1)}(\underline{t}) t_{1} \\
& +p_{a_{i} b_{j}, a_{i+1} b_{j+1}^{c}}\left(\phi_{c-1, \Lambda_{1}, \Lambda_{2}}^{\left(i+1, v_{2}^{j}\right)}(\underline{t}) v_{1}^{j}+\phi_{c-1, \Lambda_{1}}^{\left(i+1, b_{j+1}^{c}\right)}(\underline{t})\left(1-v_{1}^{j}\right)\right) t_{1} \\
& +p_{a_{i} b_{j}, a_{i+1}^{c} b_{j+1}}\left(\phi_{c-1, \Lambda_{1}, \Lambda_{2}}^{\left(u_{i}^{i}, j+1\right)}(\underline{t}) u_{1}^{i}+\phi_{c-1, \Lambda_{1}}^{\left(a_{i+1}^{c}, j+1\right)}(\underline{t})\left(1-u_{1}^{i}\right)\right) \\
& +p_{a_{i} b_{j}, a_{i+1}^{c} b b_{j+1}^{c}}\left(\phi_{c-1, \Lambda_{1}, \Lambda_{2}}^{\left(u_{i}^{i}, v_{j}^{j}\right)}(\underline{t}) u_{1}^{i} v_{1}^{j}\right. \\
& +\phi_{c-1, \Lambda_{1}}^{\left(u_{i}^{i}, b_{+1}^{c}\right)}(\underline{t}) u_{1}^{i}\left(1-v_{1}^{j}\right) \\
& +\phi_{c-1, \Lambda_{2}}^{\left(a_{i+1}^{c}, v_{2}^{j}\right)}(\underline{t})\left(1-u_{1}^{i}\right) v_{1}^{j} \\
& \left.+\phi_{c-1}^{\left(a_{i+1}^{c}, b_{j+1}^{c}\right)}(\underline{t})\left(1-u_{1}^{i}\right)\left(1-v_{1}^{j}\right)\right)
\end{aligned}
$$

if $i=k_{1}-1 ; j=1,2, \cdots, k_{2}-2$ 


$$
\begin{aligned}
& \phi_{c, \Lambda_{1}, \Lambda_{2}}^{(i, j)}(\underline{t})=p_{a_{i} b_{j}, a_{1} b_{j+1}} \phi_{c-1, \Lambda_{1}, \Lambda_{2}}^{(1, j+1)}(\underline{t}) \\
& +p_{a_{i} b_{j}, a_{1} b_{j+1}^{c}}\left(\phi_{c-1, \Lambda_{1}, \Lambda_{2}}^{\left(1, v_{2}^{j}\right)}(\underline{t}) v_{1}^{j}+\phi_{c-1, \Lambda_{1}}^{\left(1, b_{j+1}^{c}\right)}(\underline{t})\left(1-v_{1}^{j}\right)\right) \\
& +p_{a_{i} b_{j}, a_{1}^{c} b_{j+1}} \phi_{c-1, \Lambda_{2}}^{\left(a_{1}^{c}, j+1\right)}(\underline{t}) \\
& +p_{a_{i} b_{j}, a_{1}^{c} b_{j+1}^{c}}\left(\phi_{c-1, \Lambda_{2}}^{\left(a_{1}^{c}, v_{2}^{j}\right)}(\underline{t}) v_{1}^{j}+\phi_{c-1}^{\left(a_{1}^{c}, b_{j+1}^{c}\right)}(\underline{t})\left(1-v_{1}^{j}\right)\right) \\
& \text { if } i=k_{1} ; j=1,2, \cdots, k_{2}-2 \\
& \phi_{c, \Lambda_{1}, \Lambda_{2}}^{(i, j)}(\underline{t})=p_{a_{i} b_{j}, a_{i+1} b_{j+1}} \phi_{c-1, \Lambda_{1}, \Lambda_{2}}^{(i+1, j+1)}(\underline{t}) t_{2} \\
& +p_{a_{i} b_{j}, a_{i+1} b_{j+1}^{c}}\left(\phi_{c-1, \Lambda_{1}, \Lambda_{2}}^{\left(i+1, v_{2}^{j}\right)}(\underline{t}) v_{1}^{j}+\phi_{c-1, \Lambda_{1}}^{\left(i+1, b_{j+1}^{c}\right)}(\underline{t})\left(1-v_{1}^{j}\right)\right) \\
& +p_{a_{i} b_{j}, a_{i+1}^{c} b_{j+1}}\left(\phi_{c-1, \Lambda_{1}, \Lambda_{2}}^{\left(u_{u}^{i}, j+1\right)}(\underline{t}) u_{1}^{i}+\phi_{c-1, \Lambda_{1}}^{\left(a_{i+1}^{c}, j+1\right)}(\underline{t})\left(1-u_{1}^{i}\right)\right) t_{2} \\
& +p_{a_{i} b_{j}, a_{i+1}^{c} b_{j+1}^{c}}\left(\phi_{c-1, \Lambda_{1}, \Lambda_{2}}^{\left(u_{2}^{i}, v_{2}^{j}\right)}(\underline{t}) u_{1}^{i} v_{1}^{j}\right. \\
& +\phi_{c-1, \Lambda_{1}}^{\left(u_{2}^{i}, b_{j+1}^{c}\right)}(\underline{t}) u_{1}^{i}\left(1-v_{1}^{j}\right)+\phi_{c-1, \Lambda_{2}}^{\left(a_{i+1}^{c}, v_{2}^{j}\right)}(\underline{t})\left(1-u_{1}^{i}\right) v_{1}^{j} \\
& \left.+\phi_{c-1}^{\left(a_{i+1}^{c}, b_{j+1}^{c}\right)}(\underline{t})\left(1-u_{1}^{i}\right)\left(1-v_{1}^{j}\right)\right) \\
& \text { if } i=1,2, \cdots, k_{1}-2 ; j=k_{2}-1 \\
& \phi_{c, \Lambda_{1}, \Lambda_{2}}^{(i, j)}(\underline{t})=p_{a_{i} b_{j}, a_{i+1} b_{j+1}} \phi_{c-1, \Lambda_{1}, \Lambda_{2}}^{(i+1, j+1)}(\underline{t}) t_{1} t_{2} \\
& +p_{a_{i} b_{j}, a_{i+1} b_{j+1}^{c}}\left(\phi_{c-1, \Lambda_{1}, \Lambda_{2}}^{\left(i+1, v_{2}^{j}\right)}(\underline{t}) v_{1}^{j}+\phi_{c-1, \Lambda_{1}}^{\left(i+1, b_{j+1}^{c}\right)}(\underline{t})\left(1-v_{1}^{j}\right)\right) t_{1} \\
& +p_{a_{i} b_{j}, a_{i+1}^{c} b_{j+1}}\left(\phi_{c-1, \Lambda_{1}, \Lambda_{2}}^{\left(u^{i}, j+1\right)}(\underline{t}) u_{1}^{i}+\phi_{c-1, \Lambda_{1}}^{\left(a_{i+1}^{c}, j+1\right)}(\underline{t})\left(1-u_{1}^{i}\right)\right) t_{2} \\
& +p_{a_{i} b_{j}, a_{i+1}^{c} b_{j+1}^{c}}\left(\phi_{c-1, \Lambda_{1}, \Lambda_{2}}^{\left(u_{2}^{i}, v_{j}^{j}\right)}(\underline{t}) u_{1}^{i} v_{1}^{j}\right. \\
& +\phi_{c-1, \Lambda_{1}}^{\left(u_{2}^{i}, b_{j+1}^{c}\right)}(\underline{t}) u_{1}^{i}\left(1-v_{1}^{j}\right)+\phi_{c-1, \Lambda_{2}}^{\left(a_{i+1}^{c}, v_{2}^{j}\right)}(\underline{t})\left(1-u_{1}^{i}\right) v_{1}^{j} \\
& \left.+\phi_{c-1}^{\left(a_{i+1}^{c}, b_{j+1}^{c}\right)}(\underline{t})\left(1-u_{1}^{i}\right)\left(1-v_{1}^{j}\right)\right)
\end{aligned}
$$$$
\text { if } i=k_{1}-1 ; j=k_{2}-1
$$$$
\phi_{c, \Lambda_{1}, \Lambda_{2}}^{(i, j)}(\underline{t})=p_{a_{i} b_{j}, a_{i} b_{j+1}} \phi_{c-1, \Lambda_{1}, \Lambda_{2}}^{(1, j+1)}(\underline{t}) t_{2}
$$$$
+p_{a_{i} b_{j}, a_{1} b_{j+1}^{c}}\left(\phi_{c-1, \Lambda_{1}, \Lambda_{2}}^{\left(1, v_{2}^{j}\right)}(\underline{t}) v_{1}^{j}+\phi_{c-1, \Lambda_{1}}^{\left(1, b_{j+1}^{c}\right)}(\underline{t})\left(1-v_{1}^{j}\right)\right)
$$$$
+p_{a_{i} b_{j}, a_{1}^{c} b_{j+1}} \phi_{c-1, \Lambda_{2}}^{\left(a_{1}^{c}, j+1\right)}(\underline{t}) t_{2}
$$$$
+p_{a_{i} b_{j}, a_{1}^{c} b_{j+1}^{c}}\left(\phi_{c-1, \Lambda_{2}}^{\left(a_{1}^{c}, v_{2}^{j}\right)}(\underline{t}) v_{1}^{j}+\phi_{c-1}^{\left(a_{1}^{c}, b_{j+1}^{c}\right)}(\underline{t})\left(1-v_{1}^{j}\right)\right)
$$$$
\text { if } i=k_{1} ; j=k_{2}-1
$$

$$
\begin{aligned}
& \phi_{c, \Lambda_{1}, \Lambda_{2}}^{(i, j)}(\underline{t})=p_{a_{i} b_{j}, a_{i+i} b_{1}} \phi_{c-1, \Lambda_{1}, \Lambda_{2}}^{(i+1,1)}(\underline{t}) \\
& +p_{a_{i} b_{j}, a_{i+1}^{c} b_{1}}\left(\phi_{c-1, \Lambda_{1}, \Lambda_{2}}^{\left(u_{2}^{i}, 1\right)}(\underline{t}) u_{1}^{i}+\phi_{c-1, \Lambda_{2}}^{\left(a_{i+1}^{c}, 1\right)}(\underline{t})\left(1-u_{1}^{i}\right)\right) \\
& +p_{a_{i} b_{j}, a_{i+1} b_{1}^{c}} \phi_{c-1, \Lambda_{1}}^{\left(i+1, b_{i}^{c}\right)}(\underline{t}) \\
& +p_{a_{i} b_{j}, a_{i+1}^{c} b_{1}^{c}}\left(\phi_{c-1, \Lambda_{1}}^{\left(u_{i}^{i}, b_{1}^{c}\right)}(\underline{t}) u_{1}^{i}+\phi_{c-1}^{\left(a_{i+1}^{c}, b_{1}^{c}\right)}(\underline{t})\left(1-u_{1}^{i}\right)\right) \\
& \phi_{c, \Lambda_{1}, \Lambda_{2}}^{(i, j)}(\underline{t})=p_{a_{i} b_{j}, a_{i+i} b_{1}} \phi_{c-1, \Lambda_{1}, \Lambda_{2}}^{(i+1,1)}(\underline{t}) t_{1} \quad i=1,2, \cdots, k_{1}-2 ; j=k_{2} \\
& +p_{a_{i} b_{j}, a_{i+1}^{c} b_{1}}\left(\phi_{c-1, \Lambda_{1}, \Lambda_{2}}^{\left(u_{2}^{i}, 1\right)}(\underline{t}) u_{1}^{i}+\phi_{c-1, \Lambda_{2}}^{\left(a_{i+1}^{c}, 1\right)}(\underline{t})\left(1-u_{1}^{i}\right)\right) \\
& +p_{a_{i} b_{j}, a_{i+1} b_{1}^{c}} \phi_{c-1, \Lambda_{1}}^{\left(i+1, b_{i}^{c}\right)}(\underline{t}) t_{1} \quad \text { if } i=k_{1} ; j=k_{2} \\
& +p_{a_{i} b_{j}, a_{i+1}^{c} b_{1}^{c}}\left(\phi_{c-1, \Lambda_{1}}^{\left(u_{i}^{i}, b_{1}^{c}\right)}(\underline{t}) u_{1}^{i}+\phi_{c-1}^{\left(a_{i+1}^{c}, b_{1}^{c}\right)}(\underline{t})\left(1-u_{1}^{i}\right)\right) \\
& +p_{a_{i} b_{j}, a_{1} b_{1}^{c}} \phi_{c-1, \Lambda_{1}}^{\left(1, b_{1}^{c}\right)}(\underline{t})+p_{a_{i} b_{j}, a_{1}^{c} b_{1}^{c}} \phi_{c-1}^{\left(a_{1}^{c}, b_{1}^{c}\right)}(\underline{t}) \\
& \phi_{c, \Lambda_{1}, \Lambda_{2}}^{(i, j)}(\underline{t})=k_{2} \\
& p_{a_{i} b_{j}, a_{1} b_{1}} \phi_{c-1, \Lambda_{1}, \Lambda_{2}}^{(1,1)}(\underline{t})+p_{a_{i} b_{j}, a_{1}^{c} b_{1}} \phi_{c-1, \Lambda_{2}}^{\left(a_{1}^{c}, 1\right)}(\underline{t})
\end{aligned}
$$

The above system of $\left(k_{1}+2\right) \times\left(k_{2}+2\right)$ recurrent relations of conditional pgfs $\phi_{c}^{(i, j)}(\underline{t}),(i, j=0,1)$; $\phi_{c, \Lambda_{1}}^{(i, j)}(\underline{t}), \quad\left(i=0,1, \cdots, k_{1} ; j=0,1\right) ; \phi_{c, \Lambda_{2}}^{(i, j)}(\underline{t})$, $\left(i=0,1 ; j=1,2, \cdots, k_{2}\right)$ and $\phi_{c, \Lambda_{1}, \Lambda_{2}}^{(i, j)}(\underline{t})$ $\left(i=1,2, \cdots, k_{1} ; j=1,2, \cdots, k_{2}\right)$ can be written as follows.

$$
\underline{\phi}_{c}(\underline{t})= \begin{cases}\left(A+B_{1} t_{1}+B_{2} t_{2}+B_{12} t_{1} t_{2}\right) \underline{\phi}_{c-1}(\underline{t}) & \text { if } c=2,3, \cdots, n \\ \left(A+B_{1} t_{1}+B_{2} t_{2}+B_{12} t_{1} t_{2}\right) \underline{1} & \text { if } c=1\end{cases}
$$

where 1 is column vector with all its elements 1 and $\underline{\phi}_{n}(\underline{t})$ is column vector with its elements as follows.

$$
\begin{aligned}
& \left(\phi_{c}^{(0,0)} \phi_{c}^{(0,1)} \phi_{c}^{(1,0)} \phi_{c}^{(1,1)} \phi_{c, \Lambda_{1}}^{(1,0)} \ldots \phi_{c, \Lambda_{1}}^{\left(k_{1}, 1\right)} \phi_{c, \Lambda_{2}}^{(0,1)} \ldots\right. \\
& \left.\phi_{c, \Lambda_{2}}^{\left(1, k_{2}\right)} \phi_{c, \Lambda_{1}, \Lambda_{2}}^{(1,1)} \phi_{c, \Lambda_{1}, \Lambda_{2}}^{(1,2)} \cdots \phi_{c, \Lambda_{1}, \Lambda_{2}}^{\left(k_{1}, k_{2}\right)}\right)
\end{aligned}
$$

From (3.1) we get,

$$
\phi_{n}\left(t_{1}, t_{2}\right)=\underline{p}^{\prime} \underline{\phi}_{n}\left(t_{1}, t_{2}\right)
$$

where $p$ is first column of identity matrix of order $\left(k_{1}+2\right)^{\bar{x}}\left(k_{2}+2\right)$.

The recurrent use of (3.3) gives the pgf of $\left(X_{n, \Lambda_{1}}, Y_{n, \Lambda_{2}}\right)$ as follows.

$$
\phi_{n}(\underline{t})=\underline{p}^{\prime}\left(A+B_{1} t_{1}+B_{2} t_{2}+B_{12} t_{1} t_{2}\right)^{n} \underline{1}
$$




\section{Exact distribution of $\left(X_{n, k_{0}^{0}}^{0}, X_{n, k_{1}^{1}}^{1} ; Y_{n, k_{0}^{2}}^{0}, Y_{n, k_{1}^{2}}^{1}\right)$}

We note that the pgf of $\left(X_{n, \Lambda_{1}}, Y_{n, \Lambda_{2}}\right)$ is a particular case of pgf of $\left(X_{n, k_{0}^{1}}^{0}, X_{n, k_{1}^{1}}^{1} ; Y_{n, k_{0}^{2}}^{0}, Y_{n, k_{1}^{2}}^{1}\right)$. Hence we develop an algorithm to obtain the exact probability distribution of $\left(X_{n, k_{0}^{1}}^{0}, X_{n, k_{1}^{1}}^{1} ; Y_{n, k_{0}^{2}}^{0}, Y_{n, k_{1}^{2}}^{1}\right)$ which can further beapplied to obtain the exact probability distribution of $\left(X_{n, \Lambda_{1}}, Y_{n, \Lambda_{2}}\right)$ from its pgf.

Observe that the pgf of joint distribution of $\left(X_{n, k_{0}^{1}}^{0}, X_{n, k_{1}^{1}}^{1} ; Y_{n, k_{0}^{2}}^{0}, Y_{n, k_{1}^{2}}^{1}\right)$ as obtained in (2.5) in general involves matrix polynomial $P_{n}\left(t_{0}, t_{1} ; s_{0}, s_{1}\right)$ in $t_{0}, t_{1}, s_{0}$ and $s_{1}$ of order $n$ where

$$
\begin{aligned}
& P_{n}\left(t_{0}, t_{1} ; s_{0}, s_{1}\right) \\
& =\left(A+\sum_{i=0}^{1} B_{i}^{1} t_{i}+\sum_{j=0}^{1} B_{j}^{2} s_{j}+\sum_{i=0}^{1} \sum_{j=0}^{1} B_{i j}^{12} t_{i} s_{j}\right)^{n} .
\end{aligned}
$$

Hence the joint probability distribution can be obtained by expanding the polynomial with respect to $t_{0}, t_{1}, s_{0}$ and $s_{1}$.

That is,

$$
\begin{aligned}
& P\left(X_{n, k_{0}^{1}}^{0}=x_{0}, X_{n, k_{1}^{1}}^{1}=x_{1} ; Y_{n, k_{0}^{2}}^{0}=y_{0}, Y_{n, k_{1}^{2}}^{1}=y_{1}\right) \\
& =\text { coefficient of } t_{0}^{x_{0}} t_{1}^{x_{1}} s_{0}^{y_{0}} s_{1}^{y_{1}} \text { in } \phi_{n}\left(t_{0}, t_{1} ; s_{0}, s_{1}\right)
\end{aligned}
$$

i.e.

$$
\begin{aligned}
& P\left(\left(X_{n, k_{0}^{1}}^{0}, X_{n, k_{1}^{1}}^{1}\right)=\underline{x} ;\left(Y_{n, k_{0}^{2}}^{0}, Y_{n, k_{1}^{2}}^{1}\right)=\underline{y}\right) \\
& =\text { coefficient of } \underline{\underline{t}}^{\underline{\underline{x}}} \underline{\underline{S}}^{\underline{y}} \text { in } \phi_{n}\left(t_{0}, t_{1} ; s_{0}, s_{1}\right)
\end{aligned}
$$

Exact formula to obtain the coefficient matrix is quiet tedious since multiplication of matrices is not commutative operation. But the interesting recurrent relations are found between these coefficient matrices. Let $C_{n}\left(x_{0}, x_{1} ; y_{0}, y_{1}\right)=C_{n}(\underline{x} ; \underline{y})$ be the coefficient matrix of $\underline{t}^{\underline{x}} \underline{s}^{\underline{y}}$ in the expansion of matrix polynomial $P_{n}\left(t_{0}, t_{1} ; s_{0}, s_{1}\right)$ and for $n \geq 1$, let $D_{n}=\left\{(\underline{x} ; \underline{y}) \mid x_{i}, y_{i}=0,1, \cdots, n\right.$ for $\left.i=0,1\right\}$. The following Lemma gives the recurrent relations of the coefficient matrices of $C_{n}(\underline{x} ; \underline{y})$ with $C_{n-1}(\underline{x} ; \underline{y})$.

Lemma 4.1 Let $C_{n}(\underline{x} ; \underline{y})$ be the coefficient matrix of $\underline{t}^{\underline{x}} \underline{s}^{\underline{y}}$ in the expansion of the matrix polynomial $P_{n}\left(t_{0}, t_{1} ; s_{0}, s_{1}\right)$ Then $C_{n}(\underline{x} ; \underline{y})$ satisfies the following recurrent relation.

$$
\begin{aligned}
& C_{n}(\underline{x} ; \underline{y})=C_{n-1}(\underline{x} ; \underline{y}) A \\
& +\sum_{i=0}^{1} C_{n-1}\left(\underline{x}-\underline{e}_{i+1} ; \underline{y}\right) B_{i}^{1} I\left\{\underline{x}-\underline{e}_{i+1} \geq \underline{0}\right\} \\
& +\sum_{j=0}^{1} C_{n-1}\left(\underline{x} ; \underline{y}-\underline{e}_{j+1}\right) B_{j}^{2} I\left\{\underline{y}-\underline{e}_{j+1} \geq \underline{0}\right\} \\
& +\sum_{i=0}^{1} \sum_{j=0}^{1} C_{n-1}\left(\underline{x}-\underline{e}_{i+1} ; \underline{y}-\underline{e}_{j+1}\right) B_{i j}^{12} I\left\{\underline{x}^{-} \underline{e}_{i+1} \geq \underline{0} ; \underline{y}-\underline{e}_{j+1} \geq \underline{0}\right\}
\end{aligned}
$$

with $C_{1}(\underline{0} ; \underline{0})=A, \quad C_{1}\left(\underline{e}_{i+1} ; \underline{0}\right)=B_{i}^{1}, \quad C_{1}\left(\underline{0}_{\underline{e}} \underline{e}_{j+1}\right)=B_{j}^{1}$ and $C_{1}\left(\underline{e}_{i+1} ; \underline{e}_{j+1}\right)=B_{i j}^{12}$ for $i, j=0,1$. Here $\underline{e}_{i}$ is the $i^{\text {th }}$ row of the identity matrix of order 2 .

Proof Obviously for $n=1$, we have,

$$
C_{1}(\underline{x} ; \underline{y})= \begin{cases}A & \text { if } \underline{x}=\underline{0} ; \underline{y}=\underline{0} \\ B_{i}^{1} & \text { if } \underline{x}=\underline{e}_{i+1} ; \underline{y}=\underline{0}, i=0,1 \\ B_{j}^{2} & \text { if } \underline{x}=\underline{0} ; \underline{y}=\underline{e}_{j+1}, j=0,1 \\ B_{i j}^{2} & \text { if } \underline{x}=\underline{e}_{i+1} ; \underline{y}=\underline{e}_{i+1}, i=0,1 ; j=0,1 \\ O & \text { otherwise }\end{cases}
$$

where $O$ is the null matrix of same order as that of $A$.

For $n=2$, observe that $C_{2}(\underline{x}, \underline{y})$ satisfies (4.2).

Assume that Equation (4.2) is true for some $r(2 \leq r<n)$.

Hence we have,

$$
\left(A+\sum_{i=0}^{1} B_{i}^{1} t_{i}+\sum_{j=0}^{1} B_{j}^{2} s_{j}+\sum_{i=0}^{1} \sum_{j=0}^{1} B_{i j}^{12} t_{i} s_{j}\right)^{r}=\sum_{(\underline{x}, \underline{y}) \in D_{r}} C_{r}(\underline{x}, \underline{y}) \underline{t}^{\underline{x}} \underline{s}^{\underline{y}}
$$

Then

$$
\begin{aligned}
& \left(A+\sum_{i=0}^{1} B_{i}^{1} t_{i}+\sum_{j=0}^{1} B_{j}^{2} s_{j}+\sum_{i=0}^{1} \sum_{j=0}^{1} B_{i j}^{12} t_{i} s_{j}\right)^{r+1} \\
= & \left(A+\sum_{i=0}^{1} B_{i}^{1} t_{i}+\sum_{j=0}^{1} B_{j}^{2} s_{j}+\sum_{i=0}^{1} \sum_{j=0}^{1} B_{i j}^{12} t_{i} s_{j}\right)^{r} \\
& \cdot\left(A+\sum_{i=0}^{1} B_{i}^{1} t_{i}+\sum_{j=0}^{1} B_{j}^{2} s_{j}+\sum_{i=0}^{1} \sum_{j=0}^{1} B_{i j}^{12} t_{i} s_{j}\right) \\
= & \left(\sum_{(\underline{x}, \underline{y}) \in D_{r}} C_{r}(\underline{x}, \underline{y}) \underline{t}^{\underline{x}} \underline{s}^{\underline{y}}\right) \\
& \cdot\left(A+\sum_{i=0}^{1} B_{i}^{1} t_{i}+\sum_{j=0}^{1} B_{j}^{2} s_{j}+\sum_{i=0}^{1} \sum_{j=0}^{1} B_{i j}^{12} t_{i} s_{j}\right) \\
= & \sum_{(\underline{x}, \underline{y}) \in D_{r+1}} C_{r-1}(\underline{x} ; \underline{y}) A+\sum_{i=0}^{1} C_{r-1}\left(\underline{x}^{-} \underline{e}_{i+1} ; \underline{y}\right) B_{i}^{1} I\left\{\underline{x}^{-} \underline{e}_{i+1} \geq \underline{0}\right\} \\
& +\sum_{j=0}^{1} C_{r-1}\left(\underline{x} ; \underline{y}-\underline{e}_{j+1}\right) B_{j}^{2} I\left\{\underline{y}-\underline{e}_{j+1} \geq \underline{0}\right\} \\
& +\sum_{i=0}^{1} \sum_{j=0}^{1} C_{r-1}\left(\underline{x}-\underline{e}_{i+1} ; \underline{y}-\underline{e}_{j+1}\right) \\
& \left.\left(\underline{x}-\underline{e}_{i+1} \geq \underline{0}_{j} \underline{y}_{-}-\underline{e}_{j+1} \geq \underline{0}\right\}\right\} \underline{t}^{\underline{x}} \underline{s}^{\underline{y}}
\end{aligned}
$$


Hence we get the required proof of the lemma.

Theorem 4.1 The exact probability distribution of $\left(X_{n, k_{0}^{1}}^{0}, X_{n, k_{1}^{1}}^{1} ; Y_{n, k_{0}^{2}}^{0}, Y_{n, k_{1}^{2}}^{1}\right)$ is given by,

$$
\begin{aligned}
& P\left(\left(X_{n, k_{0}^{1}}^{0}, X_{n, k_{1}^{1}}^{1}\right)=\underline{x} ;\left(Y_{n, k_{0}^{2}}^{0}, Y_{n, k_{1}^{2}}^{1}\right)=\underline{y}\right), \\
& =\underline{p}^{\prime} C_{n}(\underline{x}, \underline{y}) \underline{1}
\end{aligned}
$$

where $C_{n}(\underline{x}, \underline{y})$ is the coefficient matrix of $\underline{t}^{\underline{x}} \underline{s}^{\underline{\underline{y}}}$ in expansion of matrix polynomial $P_{n}\left(t_{0}, t_{1} ; s_{0}, s_{1}\right)$ and it satisfies (4.2)

Proof The proof follows by applying the Lemma 4.1 to matrix polynomial $P_{n}\left(t_{0}, t_{1} ; s_{0}, s_{1}\right)$ involved in the pgf of distribution of $\left(X_{n, k_{0}^{1}}^{0}, X_{n, k_{1}^{1}}^{1} Y_{n, k_{0}^{2}}^{0}, Y_{n, k_{1}^{2}}^{1}\right)$ in (2.5).

Remark 4.1 The expected number of failure-runs of length $k_{0}^{1}$ in first components of $n$ Markov dependent bivariate trials is given by,

$$
E\left(X_{n, k_{0}^{1}}^{0}\right)=\left.\frac{\mathrm{d}}{\mathrm{d} t} \phi\left(t_{0}, 1 ; 1,1\right)\right|_{t_{0}=1} .
$$

On simplifying this expression, we have,

$$
\begin{gathered}
E\left(X_{n, k_{0}^{1}}^{0}\right)=\underline{p}^{\prime} \sum_{j=1}^{n}\left(A+B_{1}^{1}+B_{0}^{2}+B_{1}^{2}+B_{01}^{12}+B_{10}^{12}+B_{11}^{12}\right)^{j-1} \\
\cdot\left(B_{0}^{1}+B_{01}^{12}\right) \underline{1}
\end{gathered}
$$

\section{Waiting Time Distributions Related to Runs and Patterns}

The exact probability distribution of $\left(X_{n, k_{0}^{1}}^{0}, X_{n, k_{1}^{1}}^{1} ; Y_{n, k_{0}^{2}}^{0}, Y_{n, k_{1}^{2}}^{1}\right)$ from its pgf given in (2.5) can be expressed as,

$$
\begin{aligned}
& P\left(\left(X_{n, k_{0}^{1}}^{0}, X_{n, k_{1}^{1}}^{1}\right)=\underline{x} ;\left(Y_{n, k_{0}^{2}}^{0}, Y_{n, k_{1}^{2}}^{1}\right)=\underline{y}\right) \\
& =\underline{p}^{\prime}\left\{C_{n-1}(\underline{x} ; \underline{y}) A+\sum_{i=0}^{1} C_{n-1}\left(\underline{x}-\underline{e}_{i+1} ; \underline{y}\right) B_{i}^{1} I\left\{\underline{x}-\underline{e}_{i+1} \geq \underline{0}\right\}\right. \\
& +\sum_{j=0}^{1} C_{n-1}\left(\underline{x} ; \underline{y}-\underline{e}_{j+1}\right) B_{j}^{2} I\left\{\underline{y}-\underline{e}_{j+1} \geq \underline{0}\right\} \\
& +\sum_{i=0}^{1} \sum_{j=0}^{1} C_{n-1}\left(\underline{x}-\underline{e}_{i+1} ; \underline{y}-\underline{e}_{j+1}\right) \\
& \left.\quad \cdot B_{i j}^{12} I\left\{\underline{x}-\underline{e}_{i+1} \geq \underline{0} ; \underline{y}-\underline{e}_{j+1} \geq \underline{0}\right\}\right\} \underline{1}
\end{aligned}
$$

The components of the above expression can be interpreted as follows.

$$
\begin{aligned}
& \underline{p}^{\prime}\left\{C_{n-1}(\underline{x} ; \underline{y}) A\right\} \underline{1} \\
& =P\left(\left\{\left(X_{n, k_{0}^{1}}^{0}, X_{n, k_{1}^{1}}^{1}\right)=\underline{x},\left(Y_{n, k_{0}^{2}}^{0}, Y_{n, k_{1}^{2}}^{1}\right)=\underline{y}\right\} ;\right. \\
& \left.\quad\left\{\left(X_{n-1, k_{0}^{1}}^{0}, X_{n-1, k_{1}^{1}}^{1}\right)=\underline{x},\left(Y_{n-1, k_{0}^{2}}^{0}, Y_{n-1, k_{1}^{2}}^{1}\right)=\underline{y}\right\}\right)
\end{aligned}
$$

For $i=0,1$,

$\underline{p}^{\prime}\left\{C_{n-1}\left(\underline{x}-\underline{e}_{i+1} ; \underline{y}\right) B_{i}^{1}\right\} \underline{1}$

$=P\left(\left\{\left(X_{n, k_{0}^{1}}^{0}, X_{n, k_{1}^{1}}^{1}\right)=\underline{x},\left(Y_{n, k_{0}^{2}}^{0}, Y_{n, k_{1}^{2}}^{1}\right)=\underline{y}\right\}\right.$;

$\left.\left\{\left(X_{n-1, k_{0}^{1}}^{0}, X_{n-1, k_{1}^{1}}^{1}\right)=\underline{x}-\underline{e}_{i+1},\left(Y_{n-1, k_{0}^{2}}^{0}, Y_{n-1, k_{1}^{2}}^{1}\right)=\underline{y}\right\}\right)$

and

$$
\begin{aligned}
& \underline{p}^{\prime}\left\{C_{n-1}\left(\underline{x} ; \underline{y}-\underline{e}_{i+1}\right) B_{i}^{2}\right\} \underline{1} \\
& =P\left(\left\{\left(X_{n, k_{0}^{1}}^{0}, X_{n, k_{1}^{1}}^{1}\right)=\underline{x},\left(Y_{n, k_{0}^{2}}^{0}, Y_{n, k_{1}^{2}}^{1}\right)=\underline{y}\right\} ;\right. \\
& \quad\left\{\left(X_{n-1, k_{0}^{1}}^{0}, X_{n-1, k_{1}^{1}}^{1}\right)=\underline{x},\left(Y_{n-1, k_{0}^{2}}^{0}, Y_{n-1, k_{1}^{2}}^{1}\right)=\underline{y}-\underline{e}_{i+1}\right\}
\end{aligned}
$$

Similarly for $i, j=0,1$,

$$
\begin{aligned}
& \underline{p}^{\prime}\left\{C_{n-1}\left(\underline{x}-\underline{e}_{i+1}, \underline{y}-\underline{e}_{j+1}\right) B_{i j}^{12}\right\} \underline{1} \\
& =P\left(\left\{\left(X_{n, k_{0}^{1}}^{0}, X_{n, k_{1}^{1}}^{1}\right)=\underline{x},\left(Y_{n, k_{0}^{2}}^{0}, Y_{n, k_{1}^{2}}^{1}\right)=\underline{y}\right\} ;\right. \\
& \left.\quad\left\{\left(X_{n-1, k_{0}^{1}}^{0}, X_{n-1, k_{1}^{1}}^{1}\right)=\underline{x}-\underline{e}_{i+1},\left(Y_{n-1, k_{0}^{2}}^{0}, Y_{n-1, k_{1}^{2}}^{1}\right)=\underline{y}-\underline{e}_{j+1}\right\}\right)
\end{aligned}
$$

so that

$$
\begin{aligned}
& P\left(\left(X_{n, k_{0}^{1}}^{0}, X_{n, k_{1}^{1}}^{1}\right)=\underline{x} ;\left(Y_{n, k_{0}^{2}}^{0}, Y_{n, k_{1}^{2}}^{1}\right)=\underline{y}\right) \\
& =P\left(\left\{\left(X_{n, k_{0}^{1}}^{0}, X_{n, k_{1}^{1}}^{1}\right)=\underline{x},\left(Y_{n, k_{0}^{2}}^{0}, Y_{n, k_{1}^{2}}^{1}\right)=\underline{y}\right\}\right. \text {; } \\
& \left.\left\{\left(X_{n-1, k_{0}^{1}}^{0}, X_{n-1, k_{1}^{1}}^{1}\right)=\underline{x},\left(Y_{n-1, k_{0}^{2}}^{0}, Y_{n-1, k_{1}^{2}}^{1}\right)=\underline{y}\right\}\right) \\
& +\sum_{i=0}^{1} P\left(\left\{\left(X_{n, k_{0}^{1}}^{0}, X_{n, k_{1}^{1}}^{1}\right)=\underline{x},\left(Y_{n, k_{0}^{2}}^{0}, Y_{n, k_{1}^{2}}^{1}\right)=\underline{y}\right\} ;\right. \\
& \left.\left\{\left(X_{n-1, k_{0}^{1}}^{0}, X_{n-1, k_{1}^{1}}^{1}\right)=\underline{x}-\underline{e}_{i+1},\left(Y_{n-1, k_{0}^{2}}^{0}, Y_{n-1, k_{1}^{2}}^{1}\right)=\underline{y}\right\}\right) \\
& +\sum_{j=0}^{1} P\left(\left\{\left(X_{n, k_{0}^{1}}^{0}, X_{n, k_{1}^{1}}^{1}\right)=\underline{x},\left(Y_{n, k_{0}^{2}}^{0}, Y_{n, k_{1}^{2}}^{1}\right)=\underline{y}\right\} ;\right. \\
& \left\{\left(X_{n-1, k_{0}^{1}}^{0}, X_{n-1, k_{1}^{1}}^{1}\right)=\underline{x},\left(Y_{n-1, k_{0}^{2}}^{0}, Y_{n-1, k_{1}^{2}}^{1}\right)=\underline{y}-\underline{e}_{j+1}\right\} \\
& +\sum_{i=0}^{1} \sum_{j=0}^{1} P\left(\left\{\left(X_{n, k_{0}^{1}}^{0}, X_{n, k_{1}^{1}}^{1}\right)=\underline{x},\left(Y_{n, k_{0}^{2}}^{0}, Y_{n, k_{1}^{2}}^{1}\right)=\underline{y}\right\} ;\right. \\
& \left.\left\{\left(X_{n-1, k_{0}^{1}}^{0}, X_{n-1, k_{1}^{1}}^{1}\right)=\underline{x}-\underline{e}_{i+1},\left(Y_{n-1, k_{0}^{2}}^{0}, Y_{n-1, k_{1}^{2}}^{1}\right)=\underline{y}-\underline{e}_{j+1}\right\}\right)
\end{aligned}
$$

The above interpretation is useful for deriving different waiting time distributions.

Let $F_{0}^{i}$ be the event that failure-run of length $k_{0}^{i}$ occur for the first time in the $i^{\text {th }}$ component of the Markov dependent bivariate trials and $F_{1}^{i}$ be the event that success-run of length $k_{1}^{i}$ occur for the first time in the $i^{\text {th }}$ component of the Markov dependent bivariate trials $i=1,2$.

Let $W_{S}$ be the waiting time for sooner occurring event between $F_{0}^{1}, F_{0}^{2} F_{1}^{1}, F_{1}^{2}$. To obtain the distri- 
bution of sooner waiting time we define the following random variables.

$W_{s, j}^{i}$ : The waiting time for sooner occurring event between $F_{0}^{1}, F_{0}^{2} F_{1}^{1}, F_{1}^{2}$ given that $F_{j}^{i}$ is the sooner event (i.e. $j$-run of length $k_{j}^{i}$ of $i^{\text {th }}$ component of bivariate trials is the sooner event) $i=1,2$ and $j=0,1$.

$W_{j}^{i}$ : The waiting time for the first occurrence of $j$-run of length $k_{j}^{i}$ in the $i^{\text {th }}$ component of the bivariate trials $\left\{\left(\begin{array}{c}X_{n} \\ Y_{n}\end{array}\right), n \geq 0\right\}, i=1,2$ and $j=0,1$.

$W_{i j}^{12}$ : The waiting time until the occurrence of both events $F_{i}^{1}$ and $F_{j}^{2}$ simultaneously, $i, j=0,1$ (i.e. $i$ run of length $k_{i}^{1}$ in the first component and $j$-run of length $k_{j}^{2}$ in the second component of the bivariate trials occur simultaneously).

Then we have,

$$
\begin{aligned}
P\left(W_{S}=m\right)= & \sum_{i=0}^{1} P\left(W_{S, i}^{1}=m\right)+\sum_{j=0}^{1} P\left(W_{S, j}^{2}=m\right) \\
& +\sum_{i=0}^{1} \sum_{j=0}^{1} P\left(W_{i j}^{12}=m\right)
\end{aligned}
$$

Let $\psi_{S}(t), \psi_{S, j}^{i}(t), \psi_{j}^{i}(t)$ and $\psi^{i j}(t)$ be the pgf of $W_{S}, W_{s, j}^{i}, W_{j}^{I}$ and $W_{k j}^{12}, i=1,2$ and $k, j=0,1$ respectively. In the next sub-section we obtain the sooner waiting time distribution.

\subsection{Sooner Waiting Time Distribution}

The probability that 0 -run of length $k_{0}^{1}$ (i.e. event $F_{0}^{1}$ ) occurs for the first time at $m^{\text {th }}$ trial given that none of the events $F_{0}^{2} F_{1}^{1}, F_{1}^{2}$ has occurred until $m^{\text {th }}$ trial (i.e. $\left.P\left(W_{S, 0}^{1}=m\right)\right)$ can be written as follows.

$$
\begin{aligned}
& P\left(W_{S, 0}^{1}=m\right) \\
= & P\left(\left(X_{m, k_{0}^{1}}^{0}, X_{m, k_{1}}^{1} ; Y_{m, k_{0}^{2}}^{0}, Y_{n, k_{1}^{2}}^{1}\right)=(1,0,0,0) ;\right. \\
& \left.\left(X_{m-1, k_{0}^{1}}^{0}, X_{m-1, k_{1}^{1}}^{1} ; Y_{m-1, k_{0}^{2}}^{0}, Y_{m-1, k_{1}^{2}}^{1}\right)=(0,0,0,0)\right) \\
= & \underline{p^{\prime}}\left\{C_{m-1}(0,0,0,0)\right\} B_{0}^{1} \underline{1} \\
= & \underline{p^{\prime}} A^{m-1} B_{0}^{1} \quad m \geq k_{0}^{1}
\end{aligned}
$$

Hence $p g f$ of $W_{S, 0}^{1}$ can be given as,

$$
\psi_{S, 0}^{1}(t)=t \underline{p}^{\prime}(I-A t)^{-1} B_{0}^{1} \underline{1} .
$$

Similarly the probability that 1 -run of length $k_{1}^{1}$ (i.e. event $F_{1}^{1}$ ) occurs for the first time at $m^{\text {th }}$ trial given that none of the events $F_{0}^{1} \quad F_{0}^{2}, F_{1}^{2}$ has occurred until $m^{\text {th }}$ trial $\left(\right.$ i.e. $\left.P\left(W_{S, 1}^{1}=m\right)\right)$ can be written as follows.

$$
\begin{aligned}
& P\left(W_{S, 1}^{1}=m\right) \\
= & P\left(\left(X_{m, k_{0}^{1}}^{0}, X_{m, k_{1}^{1}}^{1} ; Y_{m, k_{0}^{2}}^{0}, Y_{m, k_{1}^{2}}^{1}\right)=(0,1,0,0) ;\right. \\
& \left.\left(X_{m-1, k_{0}^{1}}^{0}, X_{m-1, k_{1}^{1}}^{1} ; Y_{m-1, k_{0}^{2}}^{0}, Y_{m-1, k_{1}^{2}}^{1}\right)=(0,0,0,0)\right) \\
= & \underline{p}^{\prime}\left\{C_{m}(0,1,0,0)\right\} B_{1}^{1} \underline{1} \\
= & \underline{p}^{\prime} A^{m-1} B_{1}^{1} \underline{1} \quad m \geq k_{1}^{1}
\end{aligned}
$$

Hence $p g f$ of $W_{S, 1}^{1}$ is, $\psi_{S, 1}^{1}(t)=t \underline{p}^{\prime}(I-A t)^{-1} B_{1}^{1} \underline{1}$.

Similarly $P\left(W_{S, 0}^{2}=m\right)$ and $P\left(W_{S, 1}^{2}=m\right)$ is given by,

$$
\begin{aligned}
& P\left(W_{S, 0}^{2}=m\right)=\underline{p}^{\prime} A^{m-1} B_{0}^{2} \underline{1} \\
& P\left(W_{S, 1}^{2}=m\right)=\underline{p}^{\prime} A^{m-1} B_{1}^{2} \underline{1}
\end{aligned}
$$

and pgf of $W_{S, j}^{2}$ is,

$$
\psi_{S, j}^{2}(t)=t \underline{p}^{\prime}(I-A t)^{-1} B_{j}^{2} 1, j=0,1 .
$$

Now the probability that both events $F_{i}^{1}$ and $F_{j}^{2}$ occurs simultaneously for the first time at $m^{\text {th }}$ trial (i.e. $\left.P\left(W_{i, j}^{12}=m\right)\right)$ can be written as follows.

$$
\begin{aligned}
& P\left(W_{00}^{12}=m\right)=P\left(\left(X_{m, k_{0}^{1}}^{0}, X_{m, k_{1}^{1}}^{1} ; Y_{m, k_{0}^{2}}^{0}, Y_{m, k_{1}^{2}}^{1}\right)=(1,0,1,0) ;\right. \\
& \left.\left(X_{m-1, k_{0}^{1}}^{0}, X_{m-1, k_{1}^{1}}^{1} ; Y_{m-1, k_{0}^{2}}^{0}, Y_{m-1, k_{1}^{2}}^{1}\right)=(0,0,0,0)\right) \\
& =\underline{p}^{\prime}\left\{C_{m}(0,0,0,0)\right\} B_{00}^{12} \underline{1}=\underline{p}^{\prime} A^{m-1} B_{00}^{12} \underline{1} \\
& P\left(W_{01}^{12}=m\right)=P\left(\left(X_{m, k_{0}^{1}}^{0}, X_{m, k_{1}^{1}}^{1} ; Y_{m, k_{0}^{2}}^{0}, Y_{m, k_{1}^{2}}^{1}\right)=(1,0,0,1)\right. \text {; } \\
& \left.\left(X_{m-1, k_{0}^{1}}^{0}, X_{m-1, k_{1}^{1}}^{1} ; Y_{m-1, k_{0}^{2}}^{0}, Y_{m-1, k_{1}^{2}}^{1}\right)=(0,0,0,0)\right) \\
& =\underline{p}^{\prime}\left\{C_{m}(0,0,0,0)\right\} B_{01}^{12} \underline{1}=\underline{p}^{\prime} A^{m-1} B_{01}^{12} \underline{1} \\
& P\left(W_{10}^{12}=m\right)=P\left(\left(X_{m, k_{0}^{1}}^{0}, X_{m, k_{1}^{1}}^{1} ; Y_{m, k_{0}^{2}}^{0}, Y_{m, k_{1}^{2}}^{1}\right)=(0,1,1,0)\right. \text {; } \\
& \left.\left(X_{m-1, k_{0}^{1}}^{0}, X_{m-1, k_{1}^{1}}^{1} ; Y_{m-1, k_{0}^{2}}^{0}, Y_{m-1, k_{1}^{2}}^{1}\right)=(0,0,0,0)\right) \\
& =\underline{p}^{\prime}\left\{C_{m}(0,0,0,0)\right\} B_{10}^{12} \underline{1}=\underline{p}^{\prime} A^{m-1} B_{10}^{12} \underline{1} \\
& P\left(W_{11}^{12}=m\right)=P\left(\left(X_{m, k_{0}^{1}}^{0}, X_{m, k_{1}^{1}}^{1} ; Y_{m, k_{0}^{2}}^{0}, Y_{m, k_{1}^{2}}^{1}\right)=(0,1,0,1)\right. \text {; } \\
& \left.\left(X_{m-1, k_{0}^{1}}^{0}, X_{m-1, k_{1}^{1}}^{1} ; Y_{m-1, k_{0}^{2}}^{0}, Y_{m-1, k_{1}^{2}}^{1}\right)=(0,0,0,0)\right) \\
& =\underline{p}^{\prime}\left\{C_{m}(0,0,0,0)\right\} B_{11}^{12} \underline{1}=\underline{p}^{\prime} A^{m-1} B_{11}^{12} \underline{1}
\end{aligned}
$$

In general,

$$
P\left(W_{i, j}^{12}=m\right)=\underline{p}^{\prime} A^{m-1} B_{i j}^{2} 1, \quad i, j=0,1
$$

Also pgf of $W_{i j}^{12}$ is,

$$
\psi^{i j}(t)=t \underline{p}^{\prime}(I-A t)^{-1} B_{i j}^{12} \underline{1}
$$

Hence from (5.4), the exact probability distribution of 
random variable $W_{S}$ is given by,

$$
\begin{gathered}
P\left(W_{S}=m\right)=\underline{p^{\prime}} A^{m-1}\left(\sum_{i=1}^{2} \sum_{j=0}^{1} B_{j}^{i}+\sum_{i=0}^{1} \sum_{j=0}^{1} B_{i j}^{12}\right) \underline{1}, \\
m \geq \min \left(k_{0}^{1}, k_{1}^{1}, k_{0}^{2}, k_{1}^{2}\right) .
\end{gathered}
$$

The pgf of $W_{S}$ is given by,

$$
\psi_{S}(t)=t \underline{p^{\prime}}(I-A t)^{-1}\left(\sum_{i=1}^{2} \sum_{j=0}^{1} B_{j}^{i}+\sum_{i=0}^{1} \sum_{j=0}^{1} B_{i j}^{12}\right) \underline{1}
$$

Here we note that it is quiet difficult to study the later waiting time distribution between $F_{0}^{1}, F_{0}^{2} F_{1}^{1}, F_{1}^{2}$, particularly when one is interested in studying the waiting time distribution for the later occurring event between more than two events. For the waiting time distribution of later occurring event between any two runs in the components of the bivariate trials or for the two patterns in the two components of bivariate trials the theory can be developed in general. For this we refer [13] who derive the waiting time distribution of later occurring event between the two events $F_{0}$ and $F_{1}$ where $F_{i}$ $(i=0,1)$ is the event that $i$-run of length $k_{i}$ occurs for the first time in the sequence of higher order Markov dependent BT. [13] use the joint distribution of number of occurrences of 1-runs (i.e. success-runs) of length $k_{1}$ and the number of occurrences of 0-runs (i.e. failure runs) of length $k_{0}$.

\subsection{Waiting Time Distribution for Runs}

Let $W_{r_{i}, i}^{j}$ be the waiting time for the $r_{i}^{\text {th }}$ occurrence of $i$-run of length $k_{i}^{j}$ for the $j^{\text {th }}$ component of bivariate trials, $i=0,1 ; j=1,2$. We obtain the distribution of $W_{r_{i}, i}^{j}$ using distribution of $X_{n, k_{i}^{j}}^{j}$ number of occurrences of $i$-run of length $k_{i}^{j}$ for the $j^{\text {th }}$ component of $n$ bivariate trials $i=0,1 ; j=1,2$. The $p g f$

$\phi_{n}\left(t_{0}, t_{1} ; s_{0}, s_{1}\right)$ of joint distribution of $\left(X_{n, k_{0}^{1}}^{0}, X_{n, k_{1}^{1}}^{1} ; X_{n, k_{0}^{2}}^{0}, X_{n, k_{1}^{2}}^{1}\right)$ as obtained in (2.5) is as follows.

$$
\begin{aligned}
& \phi_{n}\left(t_{0}, t_{1} ; s_{0}, s_{1}\right) \\
& =\underline{p}^{\prime}\left(A+\sum_{i=0}^{1} B_{i}^{1} t_{i}+\sum_{j=0}^{1} B_{j}^{2} s_{j}+\sum_{i=0}^{1} \sum_{j=0}^{1} B_{i j}^{12} t_{i} s_{j}\right)^{n} \underline{1}
\end{aligned}
$$

In particular pgf of marginal distribution of $X_{n, k_{0}^{1}}^{1}$ is obtained by setting $t_{1}=s_{0}=s_{1}=1$ in the $p g f$

$$
\phi_{n}\left(t_{0}, t_{1} ; s_{0}, s_{1}\right) \text { of }\left(X_{n, k_{0}^{1}, 0}^{0}, X_{n, k_{1}^{1}, 1}^{1} ; Y_{n, k_{0}^{2}, 0}^{0}, Y_{n, k_{1}^{2}, 1}^{1}\right) \text { and }
$$

is as follows.

$$
\begin{aligned}
& \phi_{n}\left(t_{0}, 1 ; 1,1\right) \\
& =\underline{p}^{\prime}\left(A+B_{0}^{1} t_{0}+B_{1}^{1}+\sum_{j=0}^{1} B_{j}^{2}+B_{00}^{12} t_{0}+B_{01}^{12} t_{0}+B_{10}^{12}+B_{11}^{12}\right)^{n} \underline{1}
\end{aligned}
$$

Let pgf of $X_{n, k_{i}^{j}}^{j}$ be $\psi_{n, i}^{j}\left(t_{i}^{j}\right)$ for $i=0,1 ; j=1,2$. Then pgf of $X_{n, k_{0}^{1}}^{1}$ in (5.7) can be expressed in a simplified form as follows.

$$
\psi_{n, 0}^{1}\left(t_{0}^{1}\right)=\phi_{n}\left(t_{0}=t_{0}^{1}, 1 ; 1,1\right)=\underline{p}^{\prime}\left(A_{0}^{1}+D_{0}^{1} t_{0}^{1}\right)^{n} \underline{1}
$$

where $A_{0}^{1}=A+B_{1}^{1}+B_{0}^{2}+B_{1}^{2}+B_{10}^{12}+B_{11}^{12}$ and $D_{0}^{1}=B_{0}^{1}+B_{00}^{12}+B_{01}^{12}$.

The exact probability distribution of $X_{n, k_{0}^{1}}^{1}$ can be obtained from Lemma 4.1 as follows.

$$
P\left(X_{n, k_{0}^{1}}^{1}=r\right)=\underline{p}^{\prime} C_{n}(r) \underline{1}
$$

where $C_{n}(r)$ is the coefficient matrix of $\left(t_{0}^{1}\right)^{r}$ in the matrix polynomial $\left(A_{0}^{1}+D_{0}^{1} t_{0}^{1}\right)^{n}$ and in general for $2 \leq m \leq n \quad C_{m}(r)$ satisfies the recurrent relation

$$
C_{m}(r)=C_{m-1}(r) A_{0}^{1}+C_{m-1}(r-1) D_{0}^{1}
$$

with $C_{1}(i)= \begin{cases}A_{0}^{1} & \text { if } i=0 \\ D_{0}^{1} & \text { if } i=1 \\ O & \text { otherwise }\end{cases}$

where $O$ is the null matrix of order same as matrix $A_{0}^{1}$ and $D_{0}^{1}$. The probability in (5.8) can be written as follows.

$$
P\left(X_{n, k_{0}^{1}}^{1}=r\right)=\underline{p}^{\prime}\left\{C_{n-1}(r) A_{0}^{1}+C_{n-1}(r-1) D_{0}^{1}\right\} \underline{1}
$$

The above components of $P\left(X_{n, k_{0}^{1}}^{1}=r\right)$ can be interpreted as follows.

$$
\begin{gathered}
\underline{p}^{\prime}\left\{C_{n-1}(r) A_{0}^{1}\right\} \underline{1}=P\left(X_{n, k_{0}^{1}}^{1}=r ; X_{n-1, k_{0}^{1}}^{1}=r\right) \\
\underline{p}^{\prime}\left\{C_{n-1}(r) D_{0}^{1}\right\} \underline{1}=P\left(X_{n, k_{0}^{1}}^{1}=r ; X_{n-1, k_{0}^{1}}^{1}=r-1\right)
\end{gathered}
$$

Now we have,

$$
P\left(W_{r_{0}, 0}^{1}=m\right)=P\left(X_{m, k_{0}^{1}}^{1}=r_{0} ; X_{m-1, k_{0}^{1}}^{1}=r_{0}-1\right)
$$
we have,

Using the pgf of $X_{n, k_{0}^{1}}^{1}$ and interpretations in (5.9)

$$
P\left(W_{r_{0}, 0}^{1}=m\right)=\underline{p^{\prime}}\left\{C_{m-1}\left(r_{0}-1\right) D_{0}^{1}\right\} \underline{1}
$$

Particularly when $r_{0}=1$, we have,

$$
P\left(W_{1,0}^{1}=m\right)=\underline{p^{\prime}}\left(A^{m-1}\right) D_{0}^{1} \underline{1}
$$

The pgf of $W_{1,0}^{1}$ is given by,

$$
\xi(s)=s \underline{p^{\prime}}\left(I-A_{0}^{1} s\right)^{-1} D_{0}^{1} \underline{1}
$$

and formula for exact probability is

$$
P\left(W_{1,0}^{1}=m\right)=\underline{p^{\prime}}\left(A_{0}^{1}\right)^{m-1} D_{0}^{1} \underline{1}, \quad m \geq k_{0}^{1} .
$$

Similarly waiting time distributions of $W_{r_{i}, i}^{j}, \quad i=0,1$ and $j=1,2$ can be obtained. 


\section{Numerical Study}

In this section we present the numerical study based on the joint distribution of patterns in the sequence of bivariate trials. We consider the sequence

$$
\left\{\left(\begin{array}{l}
X_{n} \\
Y_{n}
\end{array}\right), n \geq 0\right\} \text { of }\left\{\left(\begin{array}{l}
0 \\
0
\end{array}\right),\left(\begin{array}{l}
0 \\
1
\end{array}\right),\left(\begin{array}{l}
1 \\
0
\end{array}\right),\left(\begin{array}{l}
1 \\
1
\end{array}\right)\right\} \text {-valued Markov }
$$

dependent bivariate trials with $\pi_{00}=1$ with transition probabilities as follows.

$$
\begin{aligned}
& p_{00,00}=0.2, p_{00,01}=0.3, p_{00,10}=0.4, p_{00,11}=0.1, \\
& p_{01,00}=0.3, p_{01,01}=0.1, p_{01,10}=0.5, p_{01,11}=0.1, \\
& p_{10,00}=0.1, p_{10,01}=0.4, p_{10,10}=0.3, p_{10,11}=0.2, \\
& p_{11,00}=0.2, p_{11,01}=0.2, p_{11,10}=0.2, p_{11,11}=0.4
\end{aligned}
$$

Let $\Lambda_{1}=01$ and $\Lambda_{2}=110$. The joint distribution of $\left(X_{n, \Lambda_{1}}, Y_{n, \Lambda_{2}}\right)$ is evaluated numerically for $n=10$ using the algorithm given in Theorem 4.1. The evaluated joint pmf of $\left(X_{n, \Lambda_{1}}, Y_{n, \Lambda_{2}}\right)$ is described in the following Table 1.

\section{Application and Extension}

[14] introduced the two-dimensional engineering system consisting of a grid of $m \times n$ components arranged in $m$-rows and $n$-columns. The system and its components can be either in working or failed state. The system fails if and only if a grid of size $r \times s$ components fails. Particularly, for $m=r+1$, we can formulate the states of the components in this system as a sequence of $n$ independent bivariate trials. We assume that the $i^{\text {th }}$ component in a column is in state 1 if it is in failed state and in state 0 otherwise. For $i=1,2, \cdots, n$ we define,

$X_{i}=\left\{\begin{array}{l}1 \text { if first } r \text { components in } i^{\text {th }} \text { column are in failed state } \\ 0 \text { otherwise }\end{array}\right.$ and

$$
Y_{i}=\left\{\begin{array}{l}
1 \text { if last } r \text { components in } i^{\text {th }} \text { column are in failed state } \\
0 \text { otherwise }
\end{array}\right.
$$

The reliability of consecutive- $(r, s)$-out-of- $(r+1, n)$ :

F-Lattice system can now be obtained simply by using the joint distribution of $\left(X_{n, \Lambda_{1}}, Y_{n, \Lambda_{2}}\right)$ as,

$\mathrm{P}$ (consecutive- $(r, s)$-out-of- $(r+1, n)$ : F-Lattice system works $)=P\left(X_{n, \Lambda_{1}}=0, Y_{n, \Lambda_{2}}=0\right)$,

where $\Lambda_{1}$ is 1 -run of length $S$ in the first component and $\Lambda_{2}$ is 1-run of length $s$ in the second component of the bivariate trials.

Extending the concept of bivariate trials to multivariate trials, the joint distributions of number of occurrences

\begin{tabular}{|c|c|c|c|c|c|}
\hline \multirow{2}{*}{$X_{10, \Lambda_{1}}$} & \multicolumn{4}{|c|}{$Y_{10, \Lambda_{2}}$} & \multirow{2}{*}{ Sum } \\
\hline & 0 & 1 & 2 & 3 & \\
\hline 0 & 0.00257 & 0.00666 & 0.00179 & $3.5 \mathrm{E}-05$ & 0.01106 \\
\hline 1 & 0.04765 & 0.08133 & 0.02006 & 0.00044 & 0.14949 \\
\hline 2 & 0.16288 & 0.21551 & 0.04793 & 0.00113 & 0.42744 \\
\hline 3 & 0.14927 & 0.15937 & 0.03184 & 0.00075 & 0.34122 \\
\hline 4 & 0.03465 & 0.0294 & 0.00497 & $9.9 \mathrm{E}-05$ & 0.06912 \\
\hline 5 & 0.00101 & 0.00058 & $7.3 \mathrm{E}-05$ & $1.2 \mathrm{E}-06$ & 0.00167 \\
\hline Sum & 0.39803 & 0.49285 & 0.10666 & 0.00245 & 1.00000 \\
\hline
\end{tabular}
of patterns $\Lambda_{i}$ of length $k_{i}(i=1,2, \cdots, m)$ in the $i^{\text {th }}$ component of $m$-variate trials can be used to get the reliability of general two-dimensional consecutive$r \times s$-out-of- $(m, n):$ F-Lattice system.
Table 1. Distribution of $\left(X_{10, \Lambda_{1}}, Y_{10, \Lambda_{2}}\right)$.

The pgf $\phi_{n}\left(t_{1}, t_{2}, \cdots, t_{m}\right)=\phi_{n}(\underline{t})$ of joint distribution of $X_{n, \Lambda_{i}}^{i}, i=1,2, \cdots, m$, the number of occurrences of patterns $\Lambda_{i}$ of length $k_{i}$ in the $i^{\text {th }}$ component of $m$-variate trials obtained in general by using the method of conditional pgfs is of form,

$$
\phi_{n}(\underline{t})=\underline{p}^{\prime}\left(A+\sum_{i=1}^{n} B_{i} t_{i}+\sum_{\substack{i, j=1 \\ i<j}}^{m} B_{i j} t_{i} t_{j}+\ldots+B_{12 \ldots k} t_{1} t_{2} \ldots t_{k}\right)^{n} \underline{1}
$$

Extending the Lemma 4.1 for the pgf in (7.1) exact joint probability distribution of $X_{n, \Lambda_{i}}^{i}, i=1,2, \cdots, m$ can be obtained.

The above study of runs and patterns can be extended in another direction by generalizing the sequence of Markov dependent bivariate trials to the sequence of Markov dependent multivariate trials. In the following, we discuss briefly the method of deriving the joint distribution of $\left(Y_{n, \Lambda_{1}^{2}, k_{1}}, Y_{n, \Lambda_{2}^{2}, k_{2}}, \cdots, Y_{n, \Lambda_{r}^{2}, k_{r}}\right)$ where $Y_{n, \Lambda_{i}^{2}, k_{i}}$, $(i=1,2, \cdots, r)$ denotes the number of occurrences of two dimensional patterns of rectangular shape $\Lambda_{i}^{2}(i=1,2, \cdots, r)$ in the sequence of Markov dependent multivariate trials.

$$
\text { Let } S_{m}^{2}=\left\{\underline{X}=\left(x_{1}, x_{2}, \cdots, x_{m}\right)^{\prime} \mid x_{i}=0,1 ; i=1,2, \cdots, m\right\}
$$

so that $\#\left(S_{m}^{2}\right)=2^{m}$. Consider the sequence of $m$-variate $S_{m}^{2}$-valued Markov dependent trials $\left\{\underline{X}_{i}, i \geq 0\right\}$. Let the transition probabilities of these Markov dependent trials $\left\{\underline{X}_{i}, i \geq 0\right\}$ be,

$P\left(\underline{X}_{r}=\underline{y} \mid \underline{X}_{r-1}=\underline{x}\right)=P_{\underline{x} \underline{y}}, \underline{x}, \underline{y} \in S_{m}^{2}, r \geq 1$.

and initial probabilities be $P\left(\underline{X}_{0}=\underline{x}\right)=\pi_{x} \forall \underline{x} \in S_{m}^{2}$.

Consider the two dimensional pattern of rectangular shape, $\Lambda_{i}^{2}=\underline{a}_{i 1} \underline{a}_{i 2} \cdots \underline{a}_{i k_{i}}(i=1,2, \cdots, r)$ where $\underline{a}_{i 1}, \underline{a}_{i 2}, \cdots, \underline{a}_{i k_{i}} \in S_{m}^{2}$. Let $Y_{n, \Lambda^{2}, k_{i}},(i=1,2, \cdots, r)$ be the number of occurrences of patterns $\Lambda_{i}^{2}$ in $n$ trials 
$\underline{X}_{1}, \underline{X}_{2}, \cdots, \underline{X}_{n}$. We obtain the joint distribution of $\left(Y_{n, \Lambda_{1}^{2}, k_{1}}, Y_{n, \Lambda_{2}^{2}, k_{2}}, \cdots, Y_{n, \Lambda_{r}^{2}, k_{r}}\right)$ using the method for deriving joint distribution of number of occurrences of patterns $\Lambda_{i}(i=1,2, \cdots, r)$ in the sequence of multi-state trials as done by [11]. For this consider the following transformation of sequence of $m$-variate sequence of $S_{m}^{2}$-valued Markov dependent trials $\left\{\underline{X}_{i}, i \geq 0\right\}$ to the univariate sequence of Markov dependent trials $\left\{Z_{i}, i \geq 0\right\}$.

Define the function $g: S_{m}^{2} \rightarrow S_{m}^{10}$ such that $g(\underline{x})=\sum_{i=1}^{m} 2^{i-1} x_{i}$ where $S_{m}^{10}=\left\{g(\underline{x}) \mid \underline{x} \in S_{m}^{2}\right\}$. Hence $S_{m}^{10}=\left\{0,1,2, \cdots, 2^{m}-1\right\}$. Each $\underline{x}$ in $S_{m}^{2}$ can be treated as a $m$-digit binary number. The function $g(\underline{x})$ converts this $m$-digit binary number into a unique equivalent decimal number in $S_{m}^{10}$.

Now corresponding to the $m$-variate sequence of $S_{m}^{2}$-valued Markov dependent trials $\left\{\underline{X}_{i}, i \geq 0\right\}$, we have the univariate sequence of $S_{m}^{10}$-valued Markov dependent trials $\left\{Z_{i}, i \geq 0\right\}$ where $Z_{i}=g\left(\underline{X}_{i}\right)$. The transition probabilities for the sequence of trials $\left\{\underline{X}_{i}, i \geq 0\right\}$ and for the converted sequence of trials $\left\{Z_{i}, i \geq 0\right\}$ are related as follows.

$$
\begin{aligned}
& P\left(\underline{X}_{r}=\underline{y} \mid \underline{X}_{r-1}=\underline{x}\right)=P_{\underline{x} \underline{y}} \\
& =P\left(Z_{r}=g(\underline{y}) \mid Z_{r-1}=g(\underline{x})\right)=p_{i j} \\
& \underline{x}, \underline{y} \in S_{m}^{2}, r \geq 1
\end{aligned}
$$

where $g(\underline{x})=i$ and $g(y)=j$. Convert the pattern $\Lambda_{i}^{2}=\underline{a}_{i 1} \underline{a}_{i 2} \cdots \underline{a}_{i k_{i}} \quad(i=1,2, \cdots, r)$ into a pattern $\Lambda_{i}^{10}=b_{i 1} b_{i 2} \cdots b_{i k_{i}}$ where $b_{i j}=g\left(\underline{a}_{i j}\right)$ for $j=1,2, \cdots, k_{i}$. Now the original problem of studying the joint distribution of $\left(Y_{n, \Lambda_{1}^{2}, k_{1}}, Y_{n, \Lambda_{2}^{2}, k_{2}}, \cdots, Y_{n, \Lambda_{r}^{2}, k_{r}}\right)$ in the sequence of $S_{m}^{2}$-valued Markov dependent trials $\underline{X}_{1}, \underline{X}_{2}, \cdots, \underline{X}_{n}$ reduces to the problem of studying distribution of $\left(Y_{n, \Lambda_{1}^{10}, k_{1}}, Y_{n, \Lambda_{2}^{10}, k_{2}}, \cdots, Y_{n, \Lambda_{r}^{10}, k_{r}}\right)$ for the sequence of $S_{m}^{10}$-valued Markov dependent trials $Z_{1}, Z_{2}, \cdots, Z_{n}$. [15] obtain the joint distribution of number of occurrences of $i$-runs $(i=0,1,2, \cdots, m)$ of length $k_{i}$, while [11] obtain the joint distribution of number of occurrences of patterns in the sequence of $\{0,1, \cdots, m\}$-valued Markov dependent trials using the method of conditional pgfs. The related waiting time distributions can also be studied following the same process as in Section 5 in the case of Markov dependent multivariate trials.

\section{References}

[1] S. J. Schwager, "Run Probabilities in Sequences of
Markov-Dependent Trials," Journal of American Statistical Association, Vol. 78, No. 381, 1983, pp. 168-175. doi: $10.2307 / 2287125$

[2] N. Balakrishnan and P. S. Chan, "Start-up Demonstration Tests with Rejection of Units upon Observing $d$ Failures," Annals of Institute of Statistical Mathematics, Vol. 52, No. 1, 2000, pp. 184-196. doi:10.1023/A:1004101402897

[3] W. Feller, "An Introduction to Probability Theory and Its Applications," 3rd Edition, John Wiley \& Sons, Hoboken, 1968.

[4] K. D. Ling, "On Binomial Distributions of Order $k$," Statistics \& Probability Letters, Vol. 6, No. 4, 1988, pp. 247-250. doi:10.1016/0167-7152(88)90069-7

[5] S. Aki and K. Hirano, "Number of Success-Runs of Specified Length until Certain Stopping Time Rules and Generalized Binomial Distributions of Order k," Annals of Institute of Statistical Mathematics, Vol. 52, No. 4, 2000, pp. 767-777. doi:10.1023/A:1017585512412

[6] A. M. Mood, "The Distribution Theory of Runs," Annals of Mathematical Statistics, Vol. 11, No. 4, 1940, pp. 367-392. doi:10.1214/aoms/1177731825

[7] S. Aki and K. Hirano, "Sooner and Later Waiting Time Problems for Runs in Markov Dependent Bivariate Trials," Annals of Institute of Statistical Mathematics, Vol. 51, No. 1, 1999, pp. 17-29. doi:10.1023/A:1003874900507

[8] M. Uchida, "On Generating Functions of Waiting Time Problems for Sequence Patterns of Discrete Random Variables," Annals of Institute of Statistical Mathematics, Vol. 50, No. 4, 1998, pp. 655-671. doi:10.1023/A:1003756712643

[9] D. L. Antzoulakos, "Waiting Times for Patterns in a Sequence of Multistate Trials," Journal of Applied Probability, Vol. 38, No. 2, 2001, pp. 508-518. doi:10.1239/jap/996986759

[10] K. Inoue and S. Aki, "Generalized Waiting Time Problems Associated with Pattern in Polya's Urn Scheme," Annals of Institute of Statistical Mathematics, Vol. 54, No. 3, 2002, pp. 681-688. doi:10.1023/A:1022431631697

[11] K. S. Kotwal and R. L. Shinde, "Joint Distributions of Patterns in the Sequence of Markov Dependent MultiState Trials," 2011 Submitted.

[12] S. Aki and K. Hirano, "Waiting Time Problems for a Two-Dimensional Pattern," Annals of Institute of Statistical Mathematics, Vol. 56, No. 1, 2004, 169-182. doi:10.1007/BF02530530

[13] K. S. Kotwal and R. L. Shinde, "Joint Distributions of Runs in a Sequence of Higher-Order Two-State Markov trials," Annals of Institute of Statistical Mathematics, Vol. 58, No. 1, 2006, pp. 537-554. doi:10.1007/s10463-005-0024-6

[14] A. A. Salvia and W. C. Lasher, "2-Dimensional Consecutive k-out-of- $n$ : F Models," IEEE Transactions on Reliability, Vol. R-39, No. 3, 1990, pp. 382-385. doi: $10.1109 / 24.103023$ 
[15] R. L. Shinde and K. S. Kotwal, "On the Joint Distribution of Runs in the Sequence of Markov Dependent MultiState Trials," Statistics \& Probability Letters, Vol. 76, No.
10, 2006, pp. 1065-1074.

doi:10.1016/j.spl.2005.12.005 